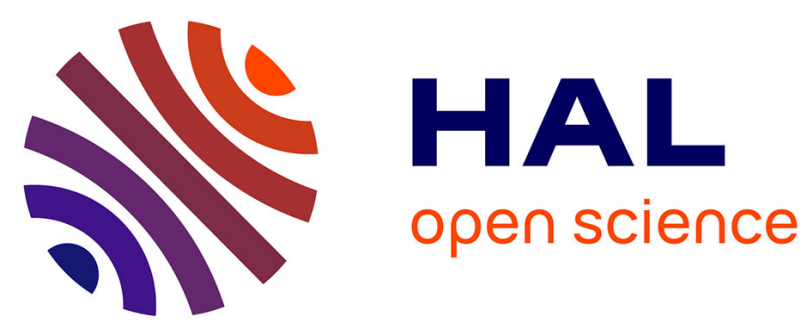

\title{
Edge Element Methods for Maxwell's Equations with Strong Convergence for Gauss' Laws
}

Patrick Ciarlet, Haijun Wu, Jun Zou

\section{To cite this version:}

Patrick Ciarlet, Haijun Wu, Jun Zou. Edge Element Methods for Maxwell's Equations with Strong Convergence for Gauss' Laws. SIAM Journal on Numerical Analysis, 2014, 52 (2), pp.779-807. 10.1137/120899856 . hal-01112201

\section{HAL Id: hal-01112201 https://hal.science/hal-01112201}

Submitted on 2 Feb 2015

HAL is a multi-disciplinary open access archive for the deposit and dissemination of scientific research documents, whether they are published or not. The documents may come from teaching and research institutions in France or abroad, or from public or private research centers.
L'archive ouverte pluridisciplinaire HAL, est destinée au dépôt et à la diffusion de documents scientifiques de niveau recherche, publiés ou non, émanant des établissements d'enseignement et de recherche français ou étrangers, des laboratoires publics ou privés. 


\title{
Edge Element Methods for Maxwell's Equations with Strong Convergence for Gauss' Laws
}

\author{
Patrick Ciarlet, Jr. ${ }^{*} \quad$ Haijun $\mathrm{Wu}^{\dagger} \quad$ Jun Zou ${ }^{\ddagger}$
}

March 20, 2014

\begin{abstract}
In this paper we propose and investigate some edge element approximations for three Maxwell systems in three dimensions: the stationary Maxwell equations, the time-harmonic Maxwell equations and the time-dependent Maxwell equations. These approximations have three novel features. First, the resulting discrete edge element systems can be solved by some existing preconditioned solvers with optimal convergence rate independent of finite element meshes, including the stationary Maxwell equations. Second, they ensure the optimal strong convergence of the Gauss' laws in some appropriate norm, in addition to the standard optimal convergence in energy-norm, under the general weak regularity assumptions that hold for both convex and non-convex polyhedral domains and for the discontinuous coefficients that may have large jumps across the interfaces between different media. Finally, no saddle-point discrete systems are needed to solve for the stationary Maxwell equations, unlike most existing edge element schemes.
\end{abstract}

Key Words. Maxwell's equations, edge elements, Gauss' laws, error estimates.

\section{Introduction}

The Nédélec's edge element methods are popular and efficient for the discretization of the Maxwell's equations, and have been extensively studied numerically and theoretically [3, 10, 16, 21, 29, 30]. But there is an interesting and important issue about the convergence of edge element methods, which has not been investigated much in the literature: how well can the Gauss' laws be satisfied by the edge element solutions? The Gauss' laws are important in many applications [5, 14, 27, 28] and should be obeyed by the finite element solutions at the discrete level. For most existing edge element methods, we know only that the Gauss' laws are satisfied in some weak sense or elementwise (e.g., the edge element solutions of first family with lowest order are divergence-free in each element), but not much is known about if the discrete Gauss' laws converge globally or to what accuracy the Gauss' laws are satisfied globally. In this work we shall fill in the gap, and propose some efficient edge element approximations which lead to strong convergence of the divergence of the edge element solution in an appropriately selected norm, under the general weak regularity assumptions that hold for both convex and non-convex polyhedral domains and for the discontinuous coefficients that may have large jumps across the interfaces between different media. Unlike the classical schemes, no saddle-point discrete systems are needed to solve for the stationary Maxwell equations. This brings in some important advantage in numerical simulations as it is much more difficult to construct efficient preconditioning-type iterative methods for saddle-point systems than for relevant positive definite systems [23, 24].

Next we shall describe three different Maxwell systems that will be investigated in this work. Let $\Omega \subset \mathbb{R}^{3}$ be a (polyhedral) domain, that is an open, connected subset of $\mathbb{R}^{3}$ with (polyhedral) connected

*POEMS Laboratory UMR CNRS-ENSTA-INRIA 7231, ENSTA ParisTech, 828, boulevard des Maréchaux, 91762 Palaiseau Cedex, France. (patrick.ciarlet@ensta-paristech.fr).

$\dagger$ Department of Mathematics, Nanjing University, Jiangsu, 210093, P.R. China. The work of HW was partially supported by the National Magnetic Confinement Fusion Science Program under grant 2011GB105003 and by the NSF of China grants (projects 11071116, 91130004). (hjw@nju.edu.cn).

${ }^{\ddagger}$ Department of Mathematics, The Chinese University of Hong Kong, Shatin, N.T., Hong Kong, China. The work of JZ was substantially supported by Hong Kong RGC grants (Projects 405110 and 404611). (zou@math. cuhk. edu.hk). 
Lipschitz boundary $\partial \Omega$. The domain $\Omega$ may not necessarily be convex, nor topologically trivial. We denote by $\mathbf{n}$ the unit outward normal vector to the boundary. Then the three Maxwell systems of our interest can be stated as follows (formulated with the electric field $\mathbf{E}$ as the only unknown, the magnetic field $\mathbf{H}$ appearing only in the initial condition [16, 29]), where $\varepsilon(\mathbf{x})$ and $\mu(\mathbf{x})$ are the dielectric constant and the magnetic permeability respectively:

\section{Stationary Maxwell equations:}

$$
\begin{aligned}
\operatorname{curl}\left(\mu^{-1} \operatorname{curl} \mathbf{E}\right)=\mathbf{f} & \text { in } \Omega, \\
\operatorname{div}(\varepsilon \mathbf{E})=\rho & \text { in } \Omega .
\end{aligned}
$$

According to (1.1), we should have $\operatorname{div} \mathbf{f}=0$ in the stationary case.

Time-harmonic Maxwell equations:

$$
\begin{aligned}
\operatorname{curl}\left(\mu^{-1} \operatorname{curl} \mathbf{E}\right)-k^{2} \varepsilon \mathbf{E}=\mathbf{f} & \text { in } \Omega, \\
\operatorname{div}(\varepsilon \mathbf{E})=\rho & \text { in } \Omega
\end{aligned}
$$

where $k>0$ is the wave number, and $k^{2}$ is assumed not to be an eigenvalue of the operator $\operatorname{curl}\left(\mu^{-1} \mathbf{c u r l}\right)$ in $\mathbf{H}_{0}(\mathbf{c u r l} ; \Omega)$ with $\varepsilon$-weighted $L^{2}$ scalar product, and it holds that $k^{2} \rho+\operatorname{div} \mathbf{f}=0$.

Time-dependent Maxwell equations:

$$
\begin{aligned}
\varepsilon \mathbf{E}_{t t}+\operatorname{curl}\left(\mu^{-1} \operatorname{curl} \mathbf{E}\right) & =\mathbf{f} \quad \text { in }(0, T) \times \Omega, \\
\operatorname{div}(\varepsilon \mathbf{E}) & =\rho \quad \text { in }(0, T) \times \Omega, \\
\mathbf{E}(0, \mathbf{x})=\mathbf{E}^{0}(\mathbf{x}), & \mathbf{E}_{t}(0, \mathbf{x})=\mathbf{F}^{0}(\mathbf{x}) \quad \text { in } \Omega
\end{aligned}
$$

where $T>0$ is the terminal time, $\rho$ is the charge density, $\mathbf{f}:=-\mathbf{J}_{t}$ and $\mathbf{J}$ is the applied current density. The initial data is $\mathbf{F}^{0}(\mathbf{x}):=\varepsilon^{-1}\left(-\mathbf{J}(0, \mathbf{x})+\mathbf{c u r l} \mathbf{H}_{0}(\mathbf{x})\right)$. Note that $\rho$ is allowed to be time-dependent. But it is assumed that $\varepsilon$ and $\mu$ are time independent, hence we have $\operatorname{div}\left(\varepsilon \mathbf{F}^{0}\right)=\rho_{t}(0, \mathbf{x})$ and the charge conservation equation $\rho_{t t}-\operatorname{div} \mathbf{f}=0$.

Boundary condition and assumptions on $\varepsilon$ and $\mu$. We shall complement the above three systems by the perfect conductor boundary condition

$$
\mathbf{E} \times \mathbf{n}=\mathbf{0} \quad \text { on } \partial \Omega,
$$

and assume that

$$
0<\varepsilon_{0} \leq \varepsilon(\mathbf{x}) \leq \varepsilon_{1}<\infty, \quad 0<\mu_{0} \leq \mu(\mathbf{x}) \leq \mu_{1}<\infty \quad \text { a.e. in } \Omega .
$$

In this paper, we shall first propose and analyse some edge element approximation for the stationary Maxwell system (1.1)-(1.2), which allows us to achieve a strong convergence for the Gauss' law. For this system, most existing finite element methods use the saddle-point formulations in order to enforce the divergence law (1.2). As it is well known, saddle-point systems are themselves much more technical and expensive to solve than their corresponding self-adjoint coercive systems, and their effective preconditioners are also more challenging to construct [23, 24, 25]. In fact the convergence behavior of the preconditioning iterative methods can be rather complicated for saddle-point systems [4,32]. This is one of the important motivations that have led us to consider the possibility to construct some edge element methods that do not involve any saddle-point systems. Indeed, as we shall see, the new method needs only to solve a symmetric and positive definite system, which is much easier to solve than the saddle-point system. In fact, optimal preconditioned solvers are available for these edge element systems, such as the Hiptmair-Xu preconditioner [22] and non-overlapping domain decomposition preconditioner [23].

Then the edge element method will be extended for both the time-harmonic Maxwell problem (1.3)(1.4) and the time-dependent Maxwell system (1.5)-(1.7) to achieve a strong convergence for the Gauss' law for the edge element solutions. This provides some new understandings to the mathematical theory of edge element methods. 
Generic notation. Throughout the paper, $C$ is used to denote a generic positive constant which is independent of the meshsize, the triangulation, (possibly) the time stepsize and the quantities/fields of interest. We also use the shorthand notation $A \lesssim B$ for the inequality $A \leq C B$, where $A$ and $B$ are two scalar quantities, and $C$ is a generic constant. We often write vector unknowns and fields in boldface. Finally, we denote by $(\cdot, \cdot)$ the usual inner product of either $L^{2}(\Omega)$ or $\mathbf{L}^{2}(\Omega):=\left(L^{2}(\Omega)\right)^{3}$, whereas $\|\cdot\|_{s}$ (respectively $|\cdot|_{s}$ ) denotes the norm (resp. semi-norm) of the Sobolev spaces $H^{s}(\Omega)$ and $\mathbf{H}^{s}(\Omega):=\left(H^{s}(\Omega)\right)^{3}$ for $s \in \mathbb{R}$.

\section{Edge and nodal element spaces and their interpolations}

In this section we present some finite element spaces and preliminary results for the subsequent analyses. Let $\mathcal{T}_{h}$ be a shape regular triangulation of $\Omega$ made up of tetrahedra, $\mathcal{F}_{h}$ the set of interior faces in $\mathcal{T}_{h}$, and $h_{K}$ the diameter of element $K$. The meshsize $h$ is defined by $h:=\max _{K} h_{K}$. Let $X_{h}$ be the lowest order edge element space of first family associated with $\mathcal{T}_{h}$, conforming in $\mathbf{H}_{0}$ (curl ; $\Omega$ ) (cf. [30]):

$$
X_{h}:=\left\{\mathbf{v}_{h} \in \mathbf{H}_{0}(\operatorname{curl} ; \Omega) ;\left.\quad \mathbf{v}_{h}\right|_{K}(\mathbf{x})=\mathbf{a}_{K}+\mathbf{b}_{K} \times \mathbf{x}, \mathbf{a}_{K}, \mathbf{b}_{K} \in \mathbb{R}^{3}, \forall K \in \mathcal{T}_{h}\right\} .
$$

Note that the functions in $X_{h}$ are piecewise divergence-free, that is, for any $\mathbf{v}_{h} \in X_{h}$ we have

$$
\operatorname{div}\left(\left.\mathbf{v}_{h}\right|_{K}\right)=0, \quad \forall K \in \mathcal{T}_{h} .
$$

However, a piecewise divergence-free function may have a globally large weak divergence, due to the jumps of its normal component at the interior faces between elements.

Now we introduce the linear $H^{1}$-conforming finite element space in $H_{0}^{1}(\Omega)$ :

$$
U_{h}:=\left\{\varphi_{h} \in H_{0}^{1}(\Omega) ;\left.\quad \varphi_{h}\right|_{K} \in P_{1}(K), \forall K \in \mathcal{T}_{h}\right\} .
$$

A function $\mathbf{v} \in \mathbf{L}^{2}(\Omega)$ is called discrete $\varepsilon$-divergence-free if $\left(\varepsilon \mathbf{v}, \nabla \varphi_{h}\right)=0$ for all $\varphi_{h} \in U_{h}$. We define $X_{0, h}^{\varepsilon}$ to be the edge element space consisting of all discrete $\varepsilon$-divergence-free functions:

$$
X_{0, h}^{\varepsilon}:=\left\{\mathbf{v}_{h} \in X_{h} ; \quad\left(\varepsilon \mathbf{v}_{h}, \nabla \varphi_{h}\right)=0, \forall \varphi_{h} \in U_{h}\right\} .
$$

Next we present a few important results. The first result is on a local trace inequality, whose proof follows from the trace inequality on the reference element and the scaling argument (cf. [11]). Given an element $K \in \mathcal{T}_{h},\|\cdot\|_{s, K}\left(\right.$ resp. $\left.|\cdot|_{s, K}\right)$ denotes the norm (resp. semi-norm) of the Sobolev spaces $H^{s}(K)$ and $\mathbf{H}^{s}(K):=\left(H^{s}(K)\right)^{3}$ for $s \in \mathbb{R}$.

Lemma 2.1. For $1 / 2<s \leq 1$, we have

$$
\|\varphi\|_{L^{2}(\partial K)} \lesssim h_{K}^{-1 / 2}\|\varphi\|_{0, K}+h_{K}^{s-1 / 2}|\varphi|_{s, K}, \quad \forall K \in \mathcal{T}_{h}, \varphi \in H^{s}(K) .
$$

Let $\Pi_{h}: L^{2}(\Omega) \mapsto U_{h}$ be the Scott-Zhang interpolation operator [33]. This interpolation is frequently used in a posteriori error analysis for finite element methods. It will be applied here to estimate a priori approximation errors for the divergence of $\varepsilon \mathbf{E}$. We have the following estimates for $\Pi_{h}$, which generalize the classical results for functions with low regularities.

Lemma 2.2. For $1 / 2<s \leq 1$ and $0 \leq t \leq s$, the following estimates hold:

$$
\begin{aligned}
\left\|\varphi-\Pi_{h} \varphi\right\|_{t} & \lesssim h^{s-t}\|\varphi\|_{s}, \quad \forall \varphi \in H^{s}(\Omega) \\
\sum_{f \in \mathcal{F}_{h}}\left\|\varphi-\Pi_{h} \varphi\right\|_{L^{2}(f)}^{2} & \lesssim h^{2 s-1}\|\varphi\|_{s}^{2}, \quad \forall \varphi \in H^{s}(\Omega) .
\end{aligned}
$$

Proof. The first estimate in (2.4) can be established by following the proof given in [33] and extending it to functions with low regularities. The idea is first to bound $\left\|\Pi_{h} \varphi\right\|_{0, K}$ and $\left\|\Pi_{h} \varphi\right\|_{s, K}$ with respect to $\|\varphi\|_{s, K}$, which yields

$$
\left\|\Pi_{h} \varphi\right\|_{0, K} \lesssim\|\varphi\|_{0, K}+h_{K}^{s}|\varphi|_{s, S_{K}}, \quad\left\|\Pi_{h} \varphi\right\|_{s, K} \lesssim h_{K}^{-s}\|\varphi\|_{0, K}+|\varphi|_{s, S_{K}},
$$


where $S_{K}:=\operatorname{int}\left(\cup_{K_{i}: K_{i} \cap K \neq \emptyset} K_{i}\right)$. Using the triangle inequality, one finds

$$
\left\|\varphi-\Pi_{h} \varphi\right\|_{0, K} \lesssim h_{K}^{s}|\varphi|_{s, S_{K}}, \quad\left\|\varphi-\Pi_{h} \varphi\right\|_{s, K} \lesssim|\varphi|_{s, S_{K}} .
$$

Then one concludes by interpolation; see the details in [13].

The second estimate in (2.4) follows from the first one and (2.3).

Finally, we recall the classical approximation results for the interpolation operator $\mathbf{r}_{h}$ onto the edge element space $X_{h}$ (cf. [16] or [29, Theorem 5.41]). We define, for $r \geq 0$,

$$
\mathbf{H}^{r}(\operatorname{curl} ; \Omega):=\left\{\mathbf{u} \in \mathbf{H}^{r}(\Omega) ; \quad \operatorname{curl} \mathbf{u} \in \mathbf{H}^{r}(\Omega)\right\},
$$

equipped with norm $\|\mathbf{u}\|_{\mathbf{H}^{r}(\mathbf{c u r l} ; \Omega)}:=\left(\|\mathbf{u}\|_{r}+\|\operatorname{curl} \mathbf{u}\|_{r}\right)^{1 / 2}$. Clearly, $\|\mathbf{u}\|_{\mathbf{H}(\operatorname{curl} ; \Omega)}=\|\mathbf{u}\|_{\mathbf{H}^{0}(\operatorname{curl} ; \Omega)}$.

Lemma 2.3. We have the following estimates for $1 / 2<r \leq 1$ :

1. For any $K \in \mathcal{T}_{h}$, if $\mathbf{u} \in \mathbf{H}^{r}(K)$ and $\left.\operatorname{curl} \mathbf{u}\right|_{K} \in D_{1}:=\left\{\mathbf{a}+b \mathbf{x}, \mathbf{a} \in \mathbb{R}^{3}, b \in \mathbb{R}\right\}$, then

$$
\left\|\mathbf{u}-\mathbf{r}_{h} \mathbf{u}\right\|_{0, K} \lesssim h_{K}^{r}\|\mathbf{u}\|_{r, K}+h_{K}\|\operatorname{curl} \mathbf{u}\|_{0, K} .
$$

2. If $\mathbf{u} \in \mathbf{H}^{r}(\operatorname{curl} ; \Omega)$, then

$$
\left\|\mathbf{u}-\mathbf{r}_{h} \mathbf{u}\right\|_{\mathbf{H}(\operatorname{curl} ; \Omega)} \lesssim h^{r}\|\mathbf{u}\|_{\mathbf{H}^{r}(\operatorname{curl} ; \Omega)} .
$$

\section{Some basic results on regularities and error estimates}

\subsection{Regularities with smooth coefficients and regular decompositions}

We first recall some basic results on the a priori regularity of the solution to the Poisson problem with homogeneous Dirichlet boundary condition. For given $f$, consider $z \in H_{0}^{1}(\Omega)$ satisfying

$$
\Delta z=f \quad \text { in } \Omega
$$

Then we have the following shift theorem (cf. $[18,19,26])$.

Lemma 3.1. There exists $\delta_{\max }^{D}>1 / 2$ depending only on the geometry of $\Omega$ such that for $0 \leq \delta<$ $\delta_{\max }^{D}, \delta \neq 1 / 2$ it holds

$$
\|z\|_{1+\delta} \lesssim\|f\|_{\delta-1}
$$

where $\delta_{\max }^{D}$ is called the limit regularity exponent of the Poisson problem with homogeneous Dirichlet boundary condition.

Remark 3.1. If $\Omega$ is convex, we have $\delta_{\max }^{D}>1$, and as a consequence,

$$
\|z\|_{2} \lesssim\|f\|_{0}
$$

On the other hand, if $\Omega$ is non-convex, we have $\delta_{\max }^{D}<1$.

If we define

$$
\Psi^{D}(\Omega):=\left\{\psi \in H_{0}^{1}(\Omega) ; \Delta \psi \in L^{2}(\Omega)\right\},
$$

then it follows from Lemma 3.1 that the continuous embedding $\Psi^{D}(\Omega) \subset H^{1+\delta}(\Omega)$ holds for $0<\delta<\delta_{\max }^{D}$, $\delta \leq 1$.

Similar regularity results hold for the Poisson problem with homogeneous Neumann boundary condition. Let $\delta_{\max }^{N}$ be the corresponding limit regularity exponent for this Neumann problem, then for a non-convex $\Omega$, we have $1 / 2<\delta_{\max }^{N}<1$, and (3.2) holds for the solution $z$ to (3.1). On the other hand, for a convex $\Omega$, we have $\delta_{\max }^{N}>1$ and the estimate (3.3). Thus, if we define

$$
\Psi^{N}(\Omega):=\left\{\psi \in H^{1}(\Omega) ; \Delta \psi \in L^{2}(\Omega), \frac{\partial \psi}{\partial n}=0 \text { on } \partial \Omega, \int_{\Omega} \psi d x=0\right\},
$$

then the continuous embedding $\Psi^{N}(\Omega) \subset H^{1+\delta}(\Omega)$ holds for $0<\delta<\delta_{\max }^{N}, \delta \leq 1$. 
Remark 3.2. For a three-dimensional polyhedral domain $\Omega$, it may happen that $\delta_{\max }^{D} \neq \delta_{\max }^{N}$. We shall often write $\delta_{\max }=\min \left(\delta_{\max }^{D}, \delta_{\max }^{N}\right)$ in the rest of the paper.

Next we discuss some close relations between spaces $\Psi^{D}(\Omega)$ and $\Psi^{N}(\Omega)$ and the following spaces:

$$
\mathbf{X}_{N}(\Omega):=\mathbf{H}_{0}(\operatorname{curl} ; \Omega) \cap \mathbf{H}(\operatorname{div} ; \Omega) \text { and } \mathbf{X}_{T}(\Omega):=\mathbf{H}(\operatorname{curl} ; \Omega) \cap \mathbf{H}_{0}(\operatorname{div} ; \Omega),
$$

which are endowed with their graph norms (also called the full norms). The following continuous regularsingular decompositions can be found, e.g., in [17, Theorem 3.5 with constant coefficients].

Lemma 3.2. For any $\mathbf{u} \in \mathbf{X}_{N}(\Omega)$ we can split it as

$$
\mathbf{u}=\mathbf{u}_{r e g}+\nabla \psi^{D}
$$

where $\mathbf{u}_{r e g} \in \mathbf{X}_{N}(\Omega) \cap \mathbf{H}^{1}(\Omega)$ and $\psi^{D} \in \Psi^{D}(\Omega)$ satisfy

$$
\left\|\mathbf{u}_{r e g}\right\|_{\mathbf{X}_{N}(\Omega)}+\left\|\mathbf{u}_{r e g}\right\|_{\mathbf{H}^{1}(\Omega)}+\left\|\psi^{D}\right\|_{H^{1}(\Omega)}+\left\|\Delta \psi^{D}\right\|_{L^{2}(\Omega)} \lesssim\|\mathbf{u}\|_{\mathbf{X}_{N}(\Omega)} .
$$

Similarly, for any $\mathbf{u} \in \mathbf{X}_{T}(\Omega)$ we can split it as

$$
\mathbf{u}=\mathbf{u}_{r e g}+\nabla \psi^{N}
$$

where $\mathbf{u}_{r e g} \in \mathbf{X}_{T}(\Omega) \cap \mathbf{H}^{1}(\Omega)$ and $\psi^{N} \in \Psi^{N}(\Omega)$ satisfy

$$
\left\|\mathbf{u}_{r e g}\right\|_{\mathbf{X}_{T}(\Omega)}+\left\|\mathbf{u}_{r e g}\right\|_{\mathbf{H}^{1}(\Omega)}+\left\|\psi^{N}\right\|_{H^{1}(\Omega)}+\left\|\Delta \psi^{N}\right\|_{L^{2}(\Omega)} \lesssim\|\mathbf{u}\|_{\mathbf{X}_{T}(\Omega)} .
$$

As a consequence of the above lemma, we have the following a priori regularities.

Corollary 3.1. The following continuous embeddings hold

- $\mathbf{X}_{N}(\Omega) \subset \bigcap_{0 \leq \delta<\delta_{\text {max }}^{D}, \delta \leq 1} \mathbf{H}^{\delta}(\Omega)$;

- $\mathbf{X}_{T}(\Omega) \subset \bigcap_{0 \leq \delta<\delta_{\max }^{N}, \delta \leq 1} \mathbf{H}^{\delta}(\Omega)$.

Moreover, both $\mathbf{X}_{N}(\Omega)$ and $\mathbf{X}_{T}(\Omega)$ are compact subsets of $\mathbf{L}^{2}(\Omega)$.

The above results suggest a useful measure of functions in $\mathbf{X}_{N}(\Omega)$.

Corollary 3.2. The seminorm

$$
\mathbf{u} \mapsto\left(\|\operatorname{curl} \mathbf{u}\|_{0}^{2}+\|\operatorname{div} \mathbf{u}\|_{0}^{2}\right)^{1 / 2}
$$

is a norm of $\mathbf{X}_{N}(\Omega)$, which is equivalent to its full norm.

Proof. We prove by contradiction. Assume there exists a sequence $\left(\mathbf{u}_{\ell}\right)_{\ell}$ of functions in $\mathbf{X}_{N}(\Omega)$ such that

$$
\left\|\mathbf{u}_{\ell}\right\|_{0}=1 \quad \forall \ell \quad \text { and } \quad \lim _{\ell \rightarrow \infty}\left(\left\|\operatorname{curl} \mathbf{u}_{\ell}\right\|_{0}+\left\|\operatorname{div} \mathbf{u}_{\ell}\right\|_{0}\right)=0 .
$$

The sequence $\left(\mathbf{u}_{\ell}\right)_{\ell}$ is bounded in $\mathbf{X}_{N}(\Omega)$ : thanks to the compact embedding of $\mathbf{X}_{N}(\Omega)$ into $\mathbf{L}^{2}(\Omega)$, there exists a subsequence, still denoted by $\left(\mathbf{u}_{\ell}\right)_{\ell}$, and $\mathbf{u} \in \mathbf{L}^{2}(\Omega)$ such that $\lim _{\ell \rightarrow \infty}\left\|\mathbf{u}_{\ell}-\mathbf{u}\right\|_{0}=0$. In particular, $\|\mathbf{u}\|_{0}=1$. Due to $\lim _{\ell \rightarrow \infty}\left\|\mathbf{c u r l} \mathbf{u}_{\ell}\right\|_{0}=0$, one obtains that curl $\mathbf{u}=0$ (weakly). Similarly, we have $\operatorname{div} \mathbf{u}=0$ (weakly).

If the domain $\Omega$ is topologically trivial, then the curl-free condition curl $\mathbf{u}=0$ implies the existence of a scalar potential $\phi \in H^{1}(\Omega): \mathbf{u}=\nabla \phi$; see [3, §3.3]. In addition, $\mathbf{u} \times \mathbf{n}=0$ on the connected boundary $\partial \Omega$ ensures that $\phi$ is constant on $\partial \Omega$. As a consequence, one can choose $\phi \in H_{0}^{1}(\Omega)$. On the other hand, $\operatorname{div} \mathbf{u}=0$ leads to $\Delta \phi=0$, so one concludes that $\phi=0$, hence $\mathbf{u}=0$. This contradicts the fact that $\|\mathbf{u}\|_{0}=1$.

If the domain is topologically non-trivial, we shall follow [3, §3.3] again. Assume that there exist $I$ nonintersecting manifolds, $\Sigma_{1}, \ldots, \Sigma_{I}$, with boundaries $\partial \Sigma_{i} \subset \partial \Omega$ such that $\dot{\Omega} \equiv \Omega \backslash \bigcup_{i=1}^{I} \Sigma_{i}$ is topologically trivial. We shall write the continuation operator from $L^{2}(\dot{\Omega})$ to $L^{2}(\Omega)$ or from $\mathbf{L}^{2}(\dot{\Omega})$ to $\mathbf{L}^{2}(\Omega)$ by ${ }^{\sim}$, and the jump across $\Sigma_{i}$ by $[\cdot]_{\Sigma_{i}}$ for $i=1, \cdots, I$. Noting that $\mathbf{u}$ is curl-free, there exists a scalar potential $\phi \in H^{1}(\dot{\Omega})$, with $[\phi]_{\Sigma_{i}}=C_{i}$ for $1 \leq i \leq I$ such that $\mathbf{u}=\widetilde{\nabla \phi}$ in $\Omega$. As we did before, $\mathbf{u} \times \mathbf{n}=0$ on the connected boundary $\partial \Omega$ yields $\phi=C$ on $\partial \Omega$. Because the boundaries $\partial \Sigma_{i}$ are all included in $\partial \Omega$, the jumps $[\phi]_{\Sigma_{i}}$ all vanish. To see this, one can take their trace on the boundary $\partial \Omega$ to obtain $C_{i}=C-C=0$ for $i=1, \cdots, I$. Therefore we see that $\mathbf{u}=\nabla \widetilde{\phi}$ in $\Omega$, where $\widetilde{\phi}$ is a scalar potential that belongs to $H^{1}(\Omega)$. Now we can conclude as we did for the topologically trivial case. 


\subsection{Regularities with discontinuous coefficients and regular decompositions}

In this section we revisit the results in Section 3.1 for the case with discontinuous coefficients $\varepsilon$ and $\mu$. For the aim we incorporate coefficients $\varepsilon$ and $\mu$ explicitly into the spaces $\mathbf{X}_{N}(\Omega)$ and $\mathbf{X}_{T}(\Omega)$, and define

$$
\begin{aligned}
& \mathbf{X}_{N}(\Omega, \varepsilon):=\left\{\mathbf{u} \in \mathbf{H}_{0}(\operatorname{curl} ; \Omega) ; \operatorname{div}(\varepsilon \mathbf{u}) \in L^{2}(\Omega)\right\} \\
& \mathbf{X}_{T}(\Omega, \mu):=\left\{\mathbf{u} \in \mathbf{H}(\mathbf{c u r l} ; \Omega) ; \operatorname{div}(\mu \mathbf{u}) \in L^{2}(\Omega), \mu \mathbf{u} \cdot \mathbf{n}=0 \text { on } \partial \Omega\right\} .
\end{aligned}
$$

Because of the jumps of the coefficient $\varepsilon$, the fact that $\operatorname{div}(\varepsilon \mathbf{u})$ belongs to $L^{2}(\Omega)$ does not ensure that $\operatorname{div} \mathbf{u} \in L^{2}(\Omega)$ any more, and we do not have the embedding $\mathbf{X}_{N}(\Omega, \varepsilon) \subset \mathbf{X}_{N}(\Omega)$.

Following [17], we assume that $\varepsilon$ and $\mu$ are piecewise constant over $\Omega$, namely there exists a partition $\mathcal{P}:=\left\{\Omega_{j}\right\}_{j=1}^{J}$ of $\Omega$ into $J$ polyhedral subdomains such that $\varepsilon_{j}:=\varepsilon_{\mid \Omega_{j}}$ and $\mu_{j}:=\mu_{\mid \Omega_{j}}$ are constants for $j=1, \cdots, J$. Then for $r>0$ we define

$$
\begin{aligned}
& \operatorname{PH}^{r}(\Omega):=\left\{u \in L^{2}(\Omega) ; \quad u_{\mid \Omega_{j}} \in H^{r}\left(\Omega_{j}\right), j=1, \cdots, J\right\} \quad \text { and } \quad \mathbf{P H}^{r}(\Omega):=\left(\operatorname{PH}^{r}(\Omega)\right)^{3}, \\
& \mathbf{P H}^{r}(\mathbf{c u r l} ; \Omega):=\left\{\mathbf{u} \in \mathbf{P H}^{r}(\Omega) ; \quad \operatorname{curl} \mathbf{u} \in \mathbf{P} \mathbf{H}^{r}(\Omega)\right\} .
\end{aligned}
$$

First, we consider the regularity of the solution $z \in H_{0}^{1}(\Omega)$ to the Poisson problem

$$
\operatorname{div}(\varepsilon \nabla z)=f \quad \text { in } \Omega
$$

with Dirichlet boundary condition and given $f$. We have the following general a priori estimate for the solution $z$; see, e.g., [31].

Lemma 3.3. There exists a constant $\delta_{\max }^{D}>0$ such that it holds

$$
\|z\|_{1+\delta} \lesssim\|f\|_{\delta-1}
$$

for $0 \leq \delta<\delta_{\max }^{D}, \delta \neq 1 / 2$. And the limit regularity exponent $\delta_{\max }^{D}$ depends only on the geometry of $\Omega$, the partition $\mathcal{P}$ and the values $\left\{\varepsilon_{j}\right\}_{j=1}^{J}$.

Depending on the maximal number of adjacent subdomains and the values $\left\{\varepsilon_{j}\right\}_{j=1}^{J}$, constant $\delta_{\max }^{D}$ may be arbitrarily small. We will now focus on the domain $\Omega$ of special geometries, for which one always has $\delta_{\max }^{D}>1 / 2$. In this case, we are still able to extend the analyses developed in Section 3.1. To this end, we assume the domain $\Omega$ (with its partition $\mathcal{P}$ ) has a geometry of one of the following two types (see, e.g., [31] and [17]):

(G1) Domain $\Omega$ is convex, and the maximal number of adjacent subdomains is equal to two;

(G2) There exists some $j$ such that $\partial \Omega \subset \partial \Omega_{j}$, and the maximal number of adjacent subdomains is equal to two.

Remark 3.3. The second type (G2) above includes the important case of isolated inclusions of media in the domain $\Omega$, which has wide applications, e.g., in inverse problems [1, 2].

Now we define

$$
\Psi^{D}(\Omega, \varepsilon):=\left\{\psi \in H_{0}^{1}(\Omega) ; \operatorname{div}(\varepsilon \nabla \psi) \in L^{2}(\Omega)\right\}
$$

and we know the continuous embedding $\Psi^{D}(\Omega, \varepsilon) \subset \mathrm{PH}^{1+\delta}(\Omega)$ for $0<\delta<\delta_{\max }^{D}$.

Similar results hold also for the problem with Neumann boundary condition. We define

$$
\Psi^{N}(\Omega, \mu):=\left\{\psi \in H^{1}(\Omega) ; \operatorname{div}(\mu \nabla \psi) \in L^{2}(\Omega), \mu \frac{\partial \psi}{\partial n}=0 \text { on } \partial \Omega, \int_{\Omega} \psi d x=0\right\}
$$

then the continuous embedding $\Psi^{N}(\Omega, \mu) \subset \mathrm{PH}^{1+\delta}(\Omega)$ holds for $0<\delta<\delta_{\max }^{N}$, where $\delta_{\max }^{N}>0$ is the limit regularity exponent for the Poisson problem with operator $\operatorname{div}(\mu \nabla \cdot)$ and homogeneous Neumann boundary condition. For the domain $\Omega$ with geometry of type (G1) or (G2), it is always true that $\delta_{\max }^{N}>1 / 2($ cf. $[31,17])$.

Now we recall some continuous regular-singular splittings of functions in $\mathbf{X}_{N}(\Omega, \varepsilon)$ and $\mathbf{X}_{T}(\Omega, \mu)(\mathrm{cf}$. $[17$, Theorem 3.5]). 
Lemma 3.4. For any $\mathbf{u} \in \mathbf{X}_{N}(\Omega, \varepsilon)$, we can decompose it as

$$
\mathbf{u}=\mathbf{u}_{r e g}+\nabla \psi^{D}
$$

where $\mathbf{u}_{\text {reg }} \in \mathbf{X}_{N}(\Omega, \varepsilon) \cap \mathbf{P} \mathbf{H}^{1}(\Omega)$ and $\psi^{D} \in \Psi^{D}(\Omega, \varepsilon)$ satisfy

$$
\left\|\mathbf{u}_{r e g}\right\|_{\mathbf{X}_{N}(\Omega, \varepsilon)}+\left\|\mathbf{u}_{r e g}\right\|_{\mathbf{P H}^{1}(\Omega)}+\left\|\psi^{D}\right\|_{H^{1}(\Omega)}+\left\|\operatorname{div}\left(\varepsilon \nabla \psi^{D}\right)\right\|_{L^{2}(\Omega)} \lesssim\|\mathbf{u}\|_{\mathbf{X}_{N}(\Omega, \varepsilon)} .
$$

Similarly, for any $\mathbf{u} \in \mathbf{X}_{T}(\Omega, \mu)$, we can decompose it as

$$
\mathbf{u}=\mathbf{u}_{r e g}+\nabla \psi^{N}
$$

where $\mathbf{u}_{\text {reg }} \in \mathbf{X}_{T}(\Omega, \mu) \cap \mathbf{P} \mathbf{H}^{1}(\Omega)$ and $\psi^{N} \in \Psi^{N}(\Omega, \mu)$ satisfy

$$
\left\|\mathbf{u}_{r e g}\right\|_{\mathbf{X}_{T}(\Omega, \mu)}+\left\|\mathbf{u}_{r e g}\right\|_{\mathbf{P H}^{1}(\Omega)}+\left\|\psi^{N}\right\|_{H^{1}(\Omega)}+\left\|\operatorname{div}\left(\mu \nabla \psi^{N}\right)\right\|_{L^{2}(\Omega)} \lesssim\|\mathbf{u}\|_{\mathbf{X}_{T}(\Omega, \mu)} .
$$

As a consequence of the above lemma, we have the following continuous embeddings.

Corollary 3.3. It holds that

- $\mathbf{X}_{N}(\Omega, \varepsilon) \subset \bigcap_{0 \leq \delta<\delta_{\text {max }}^{D}, \delta \leq 1} \mathbf{P H}^{\delta}(\Omega)$;

- $\mathbf{X}_{T}(\Omega, \mu) \subset \bigcap_{0 \leq \delta<\delta_{\text {max }}^{N}, \delta \leq 1} \mathbf{P H}^{\delta}(\Omega)$.

In particular, both $\mathbf{X}_{N}(\Omega, \varepsilon)$ and $\mathbf{X}_{T}(\Omega, \mu)$ are compact subsets of $\mathbf{L}^{2}(\Omega)$.

As in Corollary 3.2, using the result in Corollary 3.3 we can show the following result.

Corollary 3.4. The seminorm

$$
\mathbf{u} \mapsto\left(\|\operatorname{curl} \mathbf{u}\|_{0}^{2}+\|\operatorname{div}(\varepsilon \mathbf{u})\|_{0}^{2}\right)^{1 / 2}
$$

is a norm of $\mathbf{X}_{N}(\Omega, \varepsilon)$, which is equivalent to its full norm.

\subsection{Some classical error estimates}

For simplicity, we assume from now on that the charge $\rho$ belongs to $L^{2}(\Omega)$. For our subsequent edge element approximation of the Gauss' law, we introduce a discrete function $\chi_{h} \in U_{h}$ such that

$$
\left(\varepsilon \nabla \chi_{h}, \nabla \varphi_{h}\right)=-\left(\rho, \varphi_{h}\right), \quad \forall \varphi_{h} \in U_{h} .
$$

This is the piecewise linear finite element discretization of the Poisson problem with Dirichlet boundary condition: Find $\chi \in H_{0}^{1}(\Omega)$ such that:

$$
(\varepsilon \nabla \chi, \nabla \varphi)=-(\rho, \varphi), \quad \forall \varphi \in H_{0}^{1}(\Omega) .
$$

The following lemma states the a priori estimate of the solution $\chi$ to (3.17) and the error estimate of the finite element solution $\chi_{h}$ to (3.16) (cf. [8]).

Lemma 3.5. Suppose that $\varepsilon \in W^{1, \infty}(\Omega)$ and that $0<\delta<\delta_{\text {max }}^{D}, \delta \leq 1$. Then it hold that

$$
\|\chi\|_{1+\delta} \lesssim\|\rho\|_{\delta-1} \text { if further, } \delta \neq 1 / 2 ; \quad\left\|\chi-\chi_{h}\right\|_{1} \lesssim h^{\delta}|\chi|_{1+\delta} .
$$

Proof. By taking $\varphi=\chi$ in (3.17), we know that $\|\chi\|_{1} \lesssim\|\rho\|_{-1}$.

On the other hand, because of the regularity assumption on the permitivity $\varepsilon$, one can write

$$
\operatorname{div} \varepsilon \nabla \chi=\nabla \varepsilon \cdot \nabla \chi+\varepsilon \Delta \chi \quad \text { in } L^{2}(\Omega) .
$$

Hence $\Delta \chi=\varepsilon^{-1}(\rho-\nabla \varepsilon \cdot \nabla \chi)$, so it follows from (3.2)-(3.3) that

$$
\begin{aligned}
\|\chi\|_{1+\delta} & \lesssim\left\|\varepsilon^{-1}(\rho-\nabla \varepsilon \cdot \nabla \chi)\right\|_{\delta-1} \\
& \lesssim\left\|\varepsilon^{-1}\right\|_{L^{\infty}(\Omega)}\left(\|\rho\|_{\delta-1}+\|\varepsilon\|_{W^{1, \infty}(\Omega)}\|\chi\|_{1}\right) \\
& \lesssim\|\rho\|_{\delta-1} .
\end{aligned}
$$

This gives the first estimate in (3.18). The second estimate can be derived from the interpolation properties of $\Pi_{h}$ and Céa's Lemma [8]. 


\subsection{Interpolation properties of electric-like fields}

Given $\mathbf{j} \in \mathbf{L}^{2}(\Omega)$ and $g \in L^{2}(\Omega)$, we introduce $\mathbf{z} \in \mathbf{H}_{0}(\mathbf{c u r l} ; \Omega)$ to be the weak solution to

$$
\begin{aligned}
\operatorname{curl}\left(\mu^{-1} \operatorname{curl} \mathbf{z}\right)=\mathbf{j} & \text { in } \Omega, \\
\operatorname{div} \mathbf{z}=g & \text { in } \Omega .
\end{aligned}
$$

Noting that $\mathbf{z}$ belongs to $\mathbf{X}_{N}(\Omega)$, we have $\|\mathbf{z}\|_{0} \lesssim\|\operatorname{curl} \mathbf{z}\|_{0}+\|g\|_{0}$ by using the equivalence of norms (see the definition (3.8)). Using this estimate, it follows from (3.19) that

$$
\|\operatorname{curl} \mathbf{z}\|_{0}^{2} \leq\|\mu\|_{L^{\infty}(\Omega)}\left(\mu^{-1} \operatorname{curl} \mathbf{z}, \operatorname{curl} \mathbf{z}\right) \leq\|\mu\|_{L^{\infty}(\Omega)}\|\mathbf{j}\|_{0}\left(\|\operatorname{curl} \mathbf{z}\|_{0}+\|g\|_{0}\right) .
$$

This enables us to derive that $\|\mathbf{c u r l} \mathbf{z}\|_{0} \lesssim\|\mathbf{j}\|_{0}+\|g\|_{0}$ by using the Young's inequality. Hence we conclude that the solution to (3.19)-(3.20) satisfies

$$
\|\mathbf{z}\|_{0}+\|\operatorname{curl} \mathbf{z}\|_{0}+\|\operatorname{div} \mathbf{z}\|_{0} \lesssim\|\mathbf{j}\|_{0}+\|g\|_{0} .
$$

Lemma 3.6. Suppose $\mu^{-1} \in W^{1, \infty}(\Omega)$, then the solution $\mathbf{z}$ of (3.19)-(3.20) has the following error estimates for $1 / 2<\delta^{\prime}<\delta_{\max }, \delta^{\prime} \leq 1$ :

$$
\|\mathbf{z}\|_{\mathbf{H}^{\delta^{\prime}}(\operatorname{curl} ; \Omega)} \lesssim\|\mathbf{j}\|_{0}+\|g\|_{0} \quad \text { and } \quad\left\|\mathbf{z}-\mathbf{r}_{h} \mathbf{Z}\right\|_{\mathbf{H}(\operatorname{curl} ; \Omega)} \lesssim h^{\delta^{\prime}}\left(\|\mathbf{j}\|_{0}+\|g\|_{0}\right) .
$$

Proof. Recall that $\mathbf{X}_{N}(\Omega)$ is continuously embedded into $\mathbf{H}^{\delta^{\prime}}(\Omega)$ (cf. Corollary 3.1). So we know from (3.21) that

$$
\|\mathbf{z}\|_{\delta^{\prime}} \lesssim\|\mathbf{j}\|_{0}+\|g\|_{0} .
$$

Now considering $\mathbf{w}=\mathbf{c u r l} \mathbf{z}$, we have clearly $\mathbf{w} \in \mathbf{H}_{0}(\operatorname{div} ; \Omega)$ with $\operatorname{div} \mathbf{w}=0$. In addition,

$$
\mathbf{j}=\operatorname{curl}\left(\mu^{-1} \mathbf{w}\right)=\nabla \mu^{-1} \times \mathbf{w}+\mu^{-1} \operatorname{curl} \mathbf{w},
$$

hence curl $\mathbf{w}=\mu\left(\mathbf{j}-\nabla \mu^{-1} \times \mathbf{w}\right) \in \mathbf{L}^{2}(\Omega)$. So we know that $\mathbf{w}$ belongs to $\mathbf{X}_{T}(\Omega)$, and derive by direct estimates and using (3.21) that

$$
\|\mathbf{w}\|_{0}+\|\mathbf{c u r l} \mathbf{w}\|_{0}+\|\operatorname{div} \mathbf{w}\|_{0} \lesssim\|\mathbf{j}\|_{0}+\|g\|_{0} .
$$

Recall that $\mathbf{X}_{T}(\Omega)$ is also continuously embedded into $\mathbf{H}^{\delta^{\prime}}(\Omega)$ (cf. Corollary 3.1 ), so we have

$$
\|\operatorname{curl} \mathbf{z}\|_{\delta^{\prime}}=\|\mathbf{w}\|_{\delta^{\prime}} \lesssim\|\mathbf{j}\|_{0}+\|g\|_{0} .
$$

Now the error estimate (3.22) follows directly from Lemma 2.3 with $r=\delta^{\prime}$.

\subsection{Some properties on discrete $\varepsilon$-divergence-free functions}

The following lemma says that a discrete $\varepsilon$-divergence-free function can be well approximated by a continuous $\varepsilon$-divergence-free function. The results for $\varepsilon=1$ are well-known (cf. [29, Lemma 7.6]).

Lemma 3.7. Suppose $\varepsilon \in W^{1, \infty}(\Omega)$. For any $\mathbf{w}_{h} \in X_{0, h}^{\varepsilon}$ there exists a function $\mathbf{w}^{h} \in \mathbf{H}_{0}(\mathbf{c u r l} ; \Omega)$ satisfying

$$
\operatorname{curl}^{h}=\operatorname{curl}_{h}, \quad \operatorname{div}\left(\varepsilon \mathbf{w}^{h}\right)=0 \quad \text { in } \quad \Omega .
$$

Moreover, the following estimates hold for $1 / 2<\delta<\delta_{\max }^{D}, \delta \leq 1$ :

$$
\left\|\mathbf{w}^{h}\right\|_{\delta} \lesssim\left\|\operatorname{curl}_{h}\right\|_{0} ; \quad\left\|\mathbf{w}^{h}-\mathbf{w}_{h}\right\|_{0} \lesssim h^{\delta}\left\|\mathbf{w}^{h}\right\|_{\delta}+h\left\|\operatorname{curl}_{h}\right\|_{0} .
$$

Proof. As $\operatorname{div}\left(\varepsilon \mathbf{w}_{h}\right) \in H^{-1}(\Omega)$, there exists $\psi \in H_{0}^{1}(\Omega)$ such that $\operatorname{div}(\varepsilon \nabla \psi)=\operatorname{div}\left(\varepsilon \mathbf{w}_{h}\right)$. Let $\mathbf{w}^{h}=$ $\mathbf{w}_{h}-\nabla \psi$, then $\mathbf{w}^{h}$ belongs to $\mathbf{X}_{N}(\Omega, \varepsilon)$ and fulfills (3.23).

Next, for any $\mathbf{w} \in \mathbf{X}_{N}(\Omega, \varepsilon)$ we can write $\varepsilon \operatorname{div} \mathbf{w}+\nabla \varepsilon \cdot \mathbf{w}=\operatorname{div}(\varepsilon \mathbf{w})$. Hence $\operatorname{div} \mathbf{w}=\varepsilon^{-1}(\operatorname{div}(\varepsilon \mathbf{w})-\nabla \varepsilon$. $\mathbf{w}) \in L^{2}(\Omega)$ because of the regularity of $\varepsilon$. This implies the embedding $\mathbf{X}_{N}(\Omega, \varepsilon) \subset \mathbf{X}_{N}(\Omega)$. Therefore, the first estimate in (3.24) follows from Corollary 3.1 and the equivalence of norms in $\mathbf{X}_{N}(\Omega, \varepsilon)$ (cf. Corollary 3.4). 
On the other hand, we have by following the proof of Lemma 7.6 in [29] that

$$
\left(\varepsilon\left(\mathbf{w}^{h}-\mathbf{w}_{h}\right), \mathbf{w}^{h}-\mathbf{w}_{h}\right)=\left(\varepsilon\left(\mathbf{w}^{h}-\mathbf{w}_{h}\right), \mathbf{w}^{h}-\mathbf{r}_{h} \mathbf{w}^{h}\right),
$$

which implies $\left\|\mathbf{w}^{h}-\mathbf{w}_{h}\right\|_{0} \lesssim\left\|\mathbf{w}^{h}-\mathbf{r}_{h} \mathbf{w}^{h}\right\|_{0}$. Then the second estimate (3.24) follows from Lemma 2.3 (with $r=\delta$ ) and (3.23). This completes the proof of Lemma 3.7.

The next lemma shows that the divergence of a discrete $\varepsilon$-divergence-free edge element function is small in $H^{-s}(\Omega)$-norm for $1 / 2<s \leq 1$.

Lemma 3.8. Suppose $\varepsilon \in W^{1, \infty}(\Omega)$ and $\left\{\mathcal{T}_{h}\right\}$ is quasi-uniform. Then for any $\mathbf{w}_{h} \in X_{0, h}^{\varepsilon}$, the following estimate holds for $1 / 2<s \leq 1$ and $1 / 2<\delta<\delta_{\max }^{D}, \delta \leq 1$ :

$$
\left\|\operatorname{div}\left(\varepsilon \mathbf{w}_{h}\right)\right\|_{-s} \lesssim h^{s+\delta-1}\left\|\operatorname{curl} \mathbf{w}_{h}\right\|_{0} .
$$

Proof. By the definition of (weak) divergence and integration by parts on each element we have for any $\varphi \in C_{0}^{\infty}(\Omega)$ and $\varphi_{h} \in U_{h}$ that

$$
\begin{aligned}
\left\langle\operatorname{div}\left(\varepsilon \mathbf{w}_{h}\right), \varphi\right\rangle= & -\left(\varepsilon \mathbf{w}_{h}, \nabla \varphi\right)=-\left(\varepsilon \mathbf{w}_{h}, \nabla\left(\varphi-\varphi_{h}\right)\right)=-\sum_{K \in \mathcal{T}_{h}} \int_{K} \varepsilon \mathbf{w}_{h} \cdot \nabla\left(\varphi-\varphi_{h}\right) \\
= & \sum_{K \in \mathcal{T}_{h}}\left(\int_{K} \operatorname{div}\left(\varepsilon \mathbf{w}_{h}\right)\left(\varphi-\varphi_{h}\right)-\int_{\partial K} \varepsilon \mathbf{w}_{h} \cdot \mathbf{n}\left(\varphi-\varphi_{h}\right)\right) \\
= & \sum_{K \in \mathcal{T}_{h}}\left(\int_{K} \nabla \varepsilon \cdot \mathbf{w}_{h}\left(\varphi-\varphi_{h}\right)-\int_{\partial K} \varepsilon \mathbf{w}_{h} \cdot \mathbf{n}\left(\varphi-\varphi_{h}\right)\right) \\
= & \left.\sum_{K \in \mathcal{T}_{h}} \int_{K} \nabla \varepsilon \cdot \mathbf{w}_{h}\left(\varphi-\varphi_{h}\right)-\sum_{f \in \mathcal{F}_{h}} \int_{f}\left[\varepsilon \mathbf{w}_{h} \cdot \mathbf{n}\right]\right]\left(\varphi-\varphi_{h}\right) \\
\lesssim & \max _{K \in \mathcal{T}_{h}}\|\varepsilon\|_{W^{1, \infty}(K)}\left\|\mathbf{w}_{h}\right\|_{0}\left\|\varphi-\varphi_{h}\right\|_{0} \\
& +\left(\sum_{f \in \mathcal{F}_{h}} \|\left[\left[\varepsilon \mathbf{w}_{h} \cdot \mathbf{n}\right] \|_{L^{2}(f)}^{2}\right)^{1 / 2}\left(\sum_{f \in \mathcal{F}_{h}}\left\|\varphi-\varphi_{h}\right\|_{L^{2}(f)}^{2}\right)^{1 / 2}\right.
\end{aligned}
$$

where $\left[\left[\varepsilon \mathbf{w}_{h} \cdot \mathbf{n}\right]\right]$ denotes the jump of the normal component of $\varepsilon \mathbf{w}_{h}$ across the face $f$ shared by two elements $K_{1}$ and $K_{2}$ :

$$
\left[\left[\varepsilon \mathbf{w}_{h} \cdot \mathbf{n}\right]\right]=\left.\left(\varepsilon \mathbf{w}_{h}\right)\right|_{K_{1}} \cdot \mathbf{n}_{1}+\left.\left(\varepsilon \mathbf{w}_{h}\right)\right|_{K_{2}} \cdot \mathbf{n}_{2}
$$

where $\mathbf{n}_{j}$ is the unit outward normal to $\partial K_{j}$ for $j=1,2$. Choosing $\varphi_{h}=\Pi_{h} \varphi$ to be the Scott-Zhang interpolant of $\varphi$ and applying (2.4), we further derive

$$
\left|\left\langle\operatorname{div}\left(\varepsilon \mathbf{w}_{h}\right), \varphi\right\rangle\right| \lesssim h^{s}\left\|\mathbf{w}_{h}\right\|_{0}\|\varphi\|_{s}+\left(\sum_{f \in \mathcal{F}_{h}}\left\|\left[\left[\varepsilon \mathbf{w}_{h} \cdot \mathbf{n}\right]\right]\right\|_{L^{2}(f)}^{2}\right)^{1 / 2} h^{s-1 / 2}\|\varphi\|_{s},
$$

which implies that

$$
\left\|\operatorname{div}\left(\varepsilon \mathbf{w}_{h}\right)\right\|_{-s} \lesssim h^{s}\left\|\mathbf{w}_{h}\right\|_{0}+h^{s-1 / 2}\left(\sum_{f \in \mathcal{F}_{h}}\left\|\left[\left[\varepsilon \mathbf{w}_{h} \cdot \mathbf{n}\right]\right]\right\|_{L^{2}(f)}^{2}\right)^{1 / 2}
$$

Let $\mathbf{w}^{h}$ be the function from Lemma 3.7, then

$$
\left\|\mathbf{w}_{h}\right\|_{0} \leq\left\|\mathbf{w}^{h}\right\|_{0}+\left\|\mathbf{w}_{h}-\mathbf{w}^{h}\right\|_{0} \lesssim\left\|\operatorname{curl} \mathbf{w}_{h}\right\|_{0} \cdot
$$

On the other hand, we obtain from the local trace inequality (2.3) with $s=\delta$ that

$$
\begin{aligned}
\left.\sum_{f \in \mathcal{F}_{h}} \|\left[\varepsilon \mathbf{w}_{h} \cdot \mathbf{n}\right]\right] \|_{L^{2}(f)}^{2} & =\sum_{f \in \mathcal{F}_{h}}\left\|\left[\left[\varepsilon\left(\mathbf{w}_{h}-\mathbf{w}^{h}\right) \cdot \mathbf{n}\right]\right]\right\|_{L^{2}(f)}^{2} \\
& \lesssim \sum_{K \in \mathcal{T}_{h}}\left(h_{K}^{-1}\left\|\mathbf{w}_{h}-\mathbf{w}^{h}\right\|_{0, K}^{2}+h_{K}^{2 \delta-1}\left|\mathbf{w}_{h}-\mathbf{w}^{h}\right|_{\delta, K}^{2}\right) \\
& \lesssim \sum_{K \in \mathcal{T}_{h}}\left(h^{-1}\left\|\mathbf{w}_{h}-\mathbf{w}^{h}\right\|_{0, K}^{2}+h_{K}^{2 \delta-1}\left|\mathbf{w}_{h}\right|_{\delta, K}^{2}+h_{K}^{2 \delta-1}\left|\mathbf{w}^{h}\right|_{\delta, K}^{2}\right)
\end{aligned}
$$


where we used the fact that $\left\{\mathcal{T}_{h}\right\}$ is quasi-uniform for the last inequality. As $\mathbf{w}_{h}$ is piecewise divergencefree, it is easy to verify the local estimate $\left|\mathbf{w}_{h}\right|_{\delta, K} \lesssim\left|\mathbf{w}_{h}\right|_{1, K} \lesssim \|$ curl $\mathbf{w}_{h} \|_{0, K}$ on each element $K$, so we can derive from Lemma 3.7 (recall that $2 \delta-1 \leq 1$ ) that

$$
\sum_{f \in \mathcal{F}_{h}}\left\|\left[\left[\varepsilon \mathbf{w}_{h} \cdot \mathbf{n}\right]\right]\right\|_{L^{2}(f)}^{2} \lesssim h^{2 \delta-1}\left\|\operatorname{curl} \mathbf{w}_{h}\right\|_{0}^{2} .
$$

Now the desired estimate of the lemma follows from (3.26) by using (3.27) and (3.28).

Let $\chi$ and $\chi_{h}$ be the solutions to (3.17) and (3.16), respectively. Then we know $\operatorname{div}(\varepsilon \nabla \chi)=\rho$, and $\nabla \chi-\nabla \chi_{h}$ is discrete $\varepsilon$-divergence-free since

$$
\left(\varepsilon\left(\nabla \chi-\nabla \chi_{h}\right), \nabla \varphi_{h}\right)=0 \quad \forall \varphi_{h} \in U_{h} .
$$

The following lemma says that $\operatorname{div}\left(\varepsilon \nabla \chi_{h}\right)$ is a good approximation to the charge density $\rho$.

Lemma 3.9. Suppose $\varepsilon \in W^{1, \infty}(\Omega), \rho \in L^{2}(\Omega)$ and $\left\{\mathcal{T}_{h}\right\}$ is quasi-uniform. Let $\chi_{h} \in U_{h}$ be the solution to (3.16), then the following estimate holds for $1 / 2<s \leq 1$ and $1 / 2<\delta<\delta_{\max }^{D}, \delta \leq 1$ :

$$
\left\|\rho-\operatorname{div}\left(\varepsilon \nabla \chi_{h}\right)\right\|_{-s} \lesssim h^{s+\delta-1}\|\rho\|_{\delta-1} .
$$

Proof. By the definition (3.16) we can write for any $\varphi \in C_{0}^{\infty}(\Omega)$ and $\varphi_{h} \in U_{h}$ that

$$
\begin{aligned}
\left\langle\rho-\operatorname{div}\left(\varepsilon \nabla \chi_{h}\right), \varphi\right\rangle & =(\rho, \varphi)+\left(\varepsilon \nabla \chi_{h}, \nabla \varphi\right)=\left(\rho, \varphi-\varphi_{h}\right)+\left(\varepsilon \nabla \chi_{h}, \nabla\left(\varphi-\varphi_{h}\right)\right) \\
& =\left(\rho, \varphi-\varphi_{h}\right)+\sum_{K \in \mathcal{T}_{h}} \int_{K} \varepsilon \nabla \chi_{h} \cdot \nabla\left(\varphi-\varphi_{h}\right) \\
& =\left(\rho, \varphi-\varphi_{h}\right)+\sum_{K \in \mathcal{T}_{h}}\left(-\int_{K} \operatorname{div}\left(\varepsilon \nabla \chi_{h}\right)\left(\varphi-\varphi_{h}\right)+\int_{\partial K} \varepsilon \nabla \chi_{h} \cdot \mathbf{n}\left(\varphi-\varphi_{h}\right)\right) \\
& =\left(\rho, \varphi-\varphi_{h}\right)-\sum_{K \in \mathcal{T}_{h}} \int_{K} \nabla \varepsilon \cdot \nabla \chi_{h}\left(\varphi-\varphi_{h}\right)+\sum_{f \in \mathcal{F}_{h}} \int_{f}\left[\varepsilon \nabla \chi_{h} \cdot \mathbf{n}\right]\left(\varphi-\varphi_{h}\right) .
\end{aligned}
$$

Let $\varphi_{h}=\Pi_{h} \varphi$ be the Scott-Zhang interpolant of $\varphi$. Then it follows from the first (with $t=1-\delta$ ) and second estimates of (2.4) that

$$
\begin{aligned}
\left|\left\langle\rho-\operatorname{div}\left(\varepsilon \nabla \chi_{h}\right), \varphi\right\rangle\right| \lesssim & h^{s+\delta-1}\|\rho\|_{\delta-1}\|\varphi\|_{s}+h^{s} \max _{K \in \mathcal{T}_{h}}\|\varepsilon\|_{W^{1, \infty}(K)}\left\|\nabla \chi_{h}\right\|_{0}\|\varphi\|_{s} \\
& +h^{s-1 / 2}\left(\sum_{f \in \mathcal{F}_{h}}\left\|\left[\left[\varepsilon \nabla \chi_{h} \cdot \mathbf{n}\right]\right]\right\|_{L^{2}(f)}^{2}\right)^{1 / 2}\|\varphi\|_{s} .
\end{aligned}
$$

It is clear that $\left(\varepsilon \nabla \chi_{h}, \nabla \chi_{h}\right)=-\left(\rho, \chi_{h}\right) \leq\|\rho\|_{-1}\left\|\chi_{h}\right\|_{1}$. Therefore, it follows from the Poincaré's inequality that $\left\|\nabla \chi_{h}\right\|_{0} \lesssim\|\rho\|_{-1}$.

On the other hand, we obtain from the local trace inequality (2.3) with $s=\delta$ and Lemma 3.5

$$
\begin{aligned}
\left.\sum_{f \in \mathcal{F}_{h}} \|\left[\varepsilon \nabla \chi_{h} \cdot \mathbf{n}\right]\right] \|_{L^{2}(f)}^{2} & \left.=\sum_{f \in \mathcal{F}_{h}} \|\left[\varepsilon \nabla\left(\chi_{h}-\chi\right) \cdot \mathbf{n}\right]\right] \|_{L^{2}(f)}^{2} \\
& \lesssim \sum_{K \in \mathcal{T}_{h}}\left(h_{K}^{-1}\left\|\nabla\left(\chi-\chi_{h}\right)\right\|_{0, K}^{2}+h_{K}^{2 \delta-1}|\chi|_{1+\delta, K}^{2}\right) \\
& \lesssim h^{2 \delta-1}\|\rho\|_{\delta-1}^{2} .
\end{aligned}
$$

Using this we derive from (3.30) that

$$
\left|\left\langle\rho-\operatorname{div} \varepsilon \nabla \chi_{h}, \varphi\right\rangle\right| \lesssim h^{s+\delta-1}\|\rho\|_{\delta-1}\|\varphi\|_{s}, \quad \forall \varphi \in C_{0}^{\infty}(\Omega),
$$

which implies (3.29). 


\section{Stationary problem with smooth coefficients}

In this section we consider the stationary system (1.1)-(1.2) and its edge element approximation. In order to ensure that the edge element solution satisfies the divergence law (1.2), nearly all the existing edge element schemes for the system (1.1)-(1.2) need to solve a discrete saddle-point system. As it is well known, saddle-point systems are themselves much more technical and expensive to solve than their corresponding self-adjoint coercive systems, and their effective preconditioners are also more challenging to construct $[23,24,25]$. We shall propose a new edge element method that needs only to solve a symmetric and positive definite system, for which optimal preconditioned solvers are available (see Remark 4.1), and at the same time the method ensures the optimal strong convergence of the Gauss' laws in some appropriate norm.

As we shall see, the treatments are fairly different depending on whether charges are present or not.

\subsection{Charge-free case}

We first take care of the charge-free case, i.e. $\rho=0$ in (1.2), so we have $\operatorname{div} \mathbf{f}=0$ and $\operatorname{div}(\varepsilon \mathbf{E})=0$. Our idea to enforce this divergence law is simple: since $\mathbf{E}$ is $\varepsilon$-divergence-free, it is natural to require that its approximation $\mathbf{E}_{h}$ is discrete $\varepsilon$-divergence-free. We achieve this goal by adding a small perturbation $\gamma(h)(\varepsilon \mathbf{E})$ to equation (1.1). To be more precise, we define the bilinear form $a_{h}$ :

$$
a_{h}(\mathbf{u}, \mathbf{v})=\left(\mu^{-1} \operatorname{curl} \mathbf{u}, \mathbf{c u r l} \mathbf{v}\right)+\gamma(h)(\varepsilon \mathbf{u}, \mathbf{v}), \quad \forall \mathbf{u}, \mathbf{v} \in \mathbf{H}_{0}(\operatorname{curl} ; \Omega),
$$

where $\gamma(h)>0$ depends only on $h$. The parameter $\gamma(h)$ just needs to be chosen appropriately small in terms of $h$ so that the newly added perturbation term does not affect our desired convergence order for the edge element solution $\mathbf{E}_{h}$. As we shall see (cf. Theorems 4.1 and 4.2), we should take $\gamma(h)$ in the range $0<\gamma(h) \lesssim h^{2}$.

With the bilinear form $a_{h}$ in (4.1), we can formulate our discrete scheme to the system (1.1)-(1.2) and (1.8) as follows: Find $\mathbf{E}_{h} \in X_{h}$ such that

$$
a_{h}\left(\mathbf{E}_{h}, \mathbf{v}_{h}\right)=\left(\mathbf{f}, \mathbf{v}_{h}\right), \quad \forall \mathbf{v}_{h} \in X_{h} .
$$

This formulation indeed ensures that $\mathbf{E}_{h}$ is discrete $\varepsilon$-divergence-free, i.e., $\mathbf{E}_{h} \in X_{0, h}^{\varepsilon}$. One can see this by taking $\mathbf{v}_{h}=\nabla \varphi_{h}$ for any $\varphi_{h} \in U_{h}$ in (4.2) and noting that $\operatorname{div} \mathbf{f}=0$.

On the other hand, for the continuous solution $\mathbf{E}$ to (1.1)-(1.2), we can easily see that

$$
a_{h}(\mathbf{E}, \mathbf{v})=(\mathbf{f}, \mathbf{v})+\gamma(h)(\varepsilon \mathbf{E}, \mathbf{v}), \quad \forall \mathbf{v} \in \mathbf{H}_{0}(\operatorname{curl} ; \Omega) .
$$

The error estimates regarding the edge element solution to (4.2) is given in Section 4.3.

\subsection{Non-charge-free case}

The treatment of the divergence law in Section 4.1 does not work when the charge is present, namely $\operatorname{div} \mathbf{f} \neq 0$ and $\operatorname{div}(\varepsilon \mathbf{E}) \neq 0$. In order to enforce this divergence law, we propose the edge element approximation of the stationary problem (1.1)-(1.2) as follows: Find $\mathbf{E}_{h} \in X_{h}$ such that

$$
a_{h}\left(\mathbf{E}_{h}, \mathbf{v}_{h}\right)=\left(\mathbf{f}, \mathbf{v}_{h}\right)+\gamma(h)\left(\varepsilon \nabla \chi_{h}, \mathbf{v}_{h}\right), \quad \forall \mathbf{v}_{h} \in X_{h}
$$

where the bilinear form $a_{h}$ is the same as in (4.1) and $\chi_{h} \in U_{h}$ is the solution to (3.16).

By taking $\mathbf{v}_{h}=\nabla \varphi_{h}$ for any $\varphi_{h} \in U_{h}$ in (4.4), we see that

$$
\left(\varepsilon\left(\mathbf{E}_{h}-\nabla \chi_{h}\right), \nabla \varphi_{h}\right)=0 \quad \forall \varphi_{h} \in U_{h},
$$

that is, $\mathbf{E}_{h}-\nabla \chi_{h} \in X_{0, h}^{\varepsilon}$, i.e. $\mathbf{E}_{h}-\nabla \chi_{h}$ is discrete $\varepsilon$-divergence-free. As we will show, $\operatorname{div}\left(\varepsilon \mathbf{E}_{h}\right)-$ $\operatorname{div}\left(\varepsilon \nabla \chi_{h}\right)$ is small in some sense. We remark that a piecewise non-divergence-free function may have a good approximation by a piecewise divergence-free function in some appropriate norm (cf. Lemma 3.9).

We shall first develop the error estimates of the edge element schemes (4.2) and (4.4) for smooth coefficients $\varepsilon$ and $\mu$, namely $\varepsilon, \mu^{-1} \in W^{1, \infty}(\Omega)$, in Subsections 4.3-4.4. Then we will handle the discontinuous coefficients $\varepsilon$ and $\mu$ in Section 5 , which will require more specific and delicate regularity results. 
For the purposes, it is natural to consider the following $\omega$-weighted $L^{2}$-norm for a given positive function $\omega \in L^{\infty}(\Omega)$ and the mesh-dependent energy norm:

$$
\begin{gathered}
\|u\|_{0, \omega}:=\left\|\omega^{1 / 2} u\right\|_{0} \forall u \in L^{2}(\Omega), \\
\|\mathbf{v}\|_{a_{h}}:=a_{h}(\mathbf{v}, \mathbf{v})^{1 / 2}=\left\{\|\mathbf{c u r l} \mathbf{v}\|_{0, \mu^{-1}}^{2}+\gamma(h)\|\mathbf{v}\|_{0, \varepsilon}^{2}\right\}^{1 / 2} .
\end{gathered}
$$

Remark 4.1. There are optimal preconditioned iterative solvers available for the edge element system (4.2) and (4.4), e.g., the Hiptmair-Xu preconditioner [22] and non-overlapping domain decomposition preconditioner [23]. In particular, if the discrete system is preconditioned by the preconditioner in [22], the resulting preconditioned system is well-conditioned, and more importantly, the condition number is independent of the parameter $\gamma(h)$; see section 8 for some numerical examples.

\subsection{Error estimates for the charge-free case}

In this section we consider the charge-free case, namely $\operatorname{div}(\varepsilon \mathbf{E})=0$. In this case we proposed the edge element scheme (4.2) with the solution $\mathbf{E}_{h}$, and will now derive the estimates for the error $\mathbf{E}-\mathbf{E}_{h}$ in the $\mathbf{H}$ (curl)-norm and for $\operatorname{div}\left(\varepsilon \mathbf{E}_{h}\right)$ in the $H^{-s}$-norm for $1 / 2<s \leq 1$.

We start with the error estimate of $\mathbf{e}:=\mathbf{E}-\mathbf{E}_{h}$ in the discrete energy norm.

Lemma 4.1. Let $\mathbf{E}$ and $\mathbf{E}_{h}$ be the solutions to (1.1)-(1.2) and (4.2), respectively. Assume that $\rho=0$, and $\gamma(h)$ is a parameter such that $0<\gamma(h) \lesssim 1$. Then it holds that

$$
\left\|\mathbf{E}-\mathbf{E}_{h}\right\|_{a_{h}} \lesssim \inf _{\mathbf{v}_{h} \in X_{h}}\left\|\mathbf{E}-\mathbf{v}_{h}\right\|_{a_{h}}+\gamma(h)^{1 / 2}\|\mathbf{E}\|_{0} .
$$

Proof. It follows from (4.2) and (4.3) that

$$
a_{h}\left(\mathbf{E}-\mathbf{E}_{h}, \mathbf{v}_{h}\right)=\gamma(h)\left(\varepsilon \mathbf{E}, \mathbf{v}_{h}\right), \quad \forall \mathbf{v}_{h} \in X_{h} .
$$

Then for any $\mathbf{v}_{h} \in X_{h}$ we derive by the Cauchy-Schwarz and Young inequalities that

$$
\begin{aligned}
\|\mathbf{e}\|_{a_{h}}^{2} & =a_{h}(\mathbf{e}, \mathbf{e})=a_{h}\left(\mathbf{e}, \mathbf{E}-\mathbf{v}_{h}\right)+a_{h}\left(\mathbf{e}, \mathbf{v}_{h}-\mathbf{E}_{h}\right)=a_{h}\left(\mathbf{e}, \mathbf{E}-\mathbf{v}_{h}\right)+\gamma(h)\left(\varepsilon \mathbf{E}, \mathbf{v}_{h}-\mathbf{E}_{h}\right) \\
& \leq\|\mathbf{e}\|_{a_{h}}\left\|\mathbf{E}-\mathbf{v}_{h}\right\|_{a_{h}}+\gamma(h)\|\mathbf{E}\|_{0, \varepsilon}\left\|\mathbf{v}_{h}-\mathbf{E}_{h}\right\|_{0, \varepsilon} \\
& \leq\|\mathbf{e}\|_{a_{h}}\left\|\mathbf{E}-\mathbf{v}_{h}\right\|_{a_{h}}+\gamma(h)\|\mathbf{E}\|_{0, \varepsilon}\left(\|\mathbf{e}\|_{0, \varepsilon}+\left\|\mathbf{E}-\mathbf{v}_{h}\right\|_{0, \varepsilon}\right) \\
& \leq\|\mathbf{e}\|_{a_{h}}\left\|\mathbf{E}-\mathbf{v}_{h}\right\|_{a_{h}}+\gamma(h)^{1 / 2}\|\mathbf{E}\|_{0, \varepsilon}\|\mathbf{e}\|_{a_{h}}+\gamma(h)\|\mathbf{E}\|_{0, \varepsilon}\left\|\mathbf{E}-\mathbf{v}_{h}\right\|_{0, \varepsilon} \\
& \leq \frac{1}{2}\|\mathbf{e}\|_{a_{h}}^{2}+\left\|\mathbf{E}-\mathbf{v}_{h}\right\|_{a_{h}}^{2}+\frac{3}{2} \gamma(h)\|\mathbf{E}\|_{0, \varepsilon}^{2}+\frac{1}{2} \gamma(h)\left\|\mathbf{E}-\mathbf{v}_{h}\right\|_{0, \varepsilon}^{2}
\end{aligned}
$$

Remark 4.2. Provided $\gamma(h)$ is bounded, it follows from the approximation property of edge element functions that

$$
\lim _{h \rightarrow 0}\left(\inf _{\mathbf{v}_{h} \in X_{h}}\left\|\mathbf{v}-\mathbf{v}_{h}\right\|_{a_{h}}\right)=0 \quad \forall \mathbf{v} \in \mathbf{H}(\operatorname{curl} ; \Omega) .
$$

Therefore, if $\gamma(h)$ is chosen such that $\lim _{h \rightarrow 0} \gamma(h)=0$ then we know from Lemma 4.1 that

$$
\lim _{h \rightarrow 0}\left\|\operatorname{curl}\left(\mathbf{E}-\mathbf{E}_{h}\right)\right\|_{0}=0 .
$$

Although the discrete energy-norm contains a weighted $L^{2}$-norm (cf. (4.7)), Lemma 4.1 does not imply the convergence in $L^{2}$-norm. We are now going to establish the $L^{2}$-norm error estimate by making use of the well-known Helmholtz decomposition and the adjoint technique.

By the Helmholtz decomposition, we can decompose $\mathbf{e}$ as $\mathbf{e}=\mathbf{E}-\mathbf{E}_{h}=\mathbf{e}_{0}+\nabla \xi$ with $\mathbf{e}_{0} \in \mathbf{L}^{2}(\Omega)$ and $\xi \in H_{0}^{1}(\Omega)$ such that

$$
\operatorname{curl} \mathbf{e}_{0}=\operatorname{curl} \mathbf{e}, \quad \operatorname{div} \mathbf{e}_{0}=0, \quad \Delta \xi=\operatorname{div} \mathbf{e},
$$


and $\left\|\mathbf{e}_{0}\right\|_{0}^{2}+\|\nabla \xi\|_{0}^{2}=\|\mathbf{e}\|_{0}^{2}$. Using this decomposition we can deduce

$$
\begin{aligned}
\|\mathbf{e}\|_{0, \varepsilon}^{2} & =\left(\varepsilon \mathbf{e}, \mathbf{e}_{0}+\nabla \xi\right)=\left(\varepsilon \mathbf{e}, \mathbf{e}_{0}\right)-\langle\operatorname{div}(\varepsilon \mathbf{e}), \xi\rangle \\
& \lesssim\|\mathbf{e}\|_{0, \varepsilon}\left\|\mathbf{e}_{0}\right\|_{0, \varepsilon}+\|\operatorname{div}(\varepsilon \mathbf{e})\|_{-1}\|\nabla \xi\|_{0}
\end{aligned}
$$

which implies

$$
\|\mathbf{e}\|_{0, \varepsilon} \lesssim\left\|\mathbf{e}_{0}\right\|_{0, \varepsilon}+\|\operatorname{div}(\varepsilon \mathbf{e})\|_{-1} .
$$

In order to estimate $\left\|\mathbf{e}_{0}\right\|_{0, \varepsilon}$, we introduce $\mathbf{z} \in \mathbf{H}_{0}(\mathbf{c u r l} ; \Omega)$ to be the weak solution to the adjoint problem (3.19)-(3.20) with $\mathbf{j}=\mathbf{e}_{0}$ and $g=0$. It follows from Lemma 3.6 that, if $\mu^{-1} \in W^{1, \infty}(\Omega)$, one has $\mathbf{z} \in \mathbf{H}^{\delta^{\prime}}(\mathbf{c u r l} ; \Omega)$ for $1 / 2<\delta^{\prime}<\delta_{\max }, \delta^{\prime} \leq 1$, with the bound $\|\mathbf{z}\|_{\mathbf{H}^{\delta^{\prime}}(\mathbf{c u r l} ; \Omega)} \lesssim\left\|\mathbf{e}_{0}\right\|_{0}$.

Using (4.8) we can write

$$
\begin{aligned}
\left\|\mathbf{e}_{0}\right\|_{0}^{2} & =\left(\mu^{-1} \operatorname{curl} \mathbf{e}_{0}, \operatorname{curl} \mathbf{z}\right)=\left(\mu^{-1} \operatorname{curl} \mathbf{e}, \operatorname{curl} \mathbf{z}\right)=a_{h}(\mathbf{e}, \mathbf{z})-\gamma(h)(\varepsilon \mathbf{e}, \mathbf{z}) \\
& =a_{h}\left(\mathbf{e}, \mathbf{z}-\mathbf{r}_{h} \mathbf{z}\right)+\gamma(h)\left(\varepsilon \mathbf{E}, \mathbf{r}_{h} \mathbf{z}\right)-\gamma(h)(\varepsilon \mathbf{e}, \mathbf{z}) .
\end{aligned}
$$

Then it follows from Lemma 2.3 that

$$
\begin{aligned}
\left\|\mathbf{e}_{0}\right\|_{0}^{2} & \leq\|\mathbf{e}\|_{a_{h}}\left\|\mathbf{z}-\mathbf{r}_{h} \mathbf{z}\right\|_{a_{h}}+\gamma(h)\|\mathbf{E}\|_{0, \varepsilon}\left\|\mathbf{r}_{h} \mathbf{z}\right\|_{0, \varepsilon}+\gamma(h)\|\mathbf{e}\|_{0, \varepsilon}\|\mathbf{z}\|_{0, \varepsilon} \\
& \lesssim\left(h^{\delta^{\prime}}\|\mathbf{e}\|_{a_{h}}+\gamma(h)\|\mathbf{E}\|_{0, \varepsilon}\right)\|\mathbf{z}\|_{\mathbf{H}^{\delta^{\prime}}(\mathbf{c u r l} ; \Omega)}+\gamma(h)^{1 / 2}\|\mathbf{e}\|_{a_{h}}\|\mathbf{z}\|_{0} \\
& \lesssim\left(h^{\delta^{\prime}}\|\mathbf{e}\|_{a_{h}}+\gamma(h)\|\mathbf{E}\|_{0, \varepsilon}\right)\left\|\mathbf{e}_{0}\right\|_{0}+\gamma(h)^{1 / 2}\|\mathbf{e}\|_{a_{h}}\left\|\mathbf{e}_{0}\right\|_{0},
\end{aligned}
$$

which implies

$$
\left\|\mathbf{e}_{0}\right\|_{0} \lesssim\left(h^{\delta^{\prime}}+\gamma(h)^{1 / 2}\right)\|\mathbf{e}\|_{a_{h}}+\gamma(h)\|\mathbf{E}\|_{0} .
$$

Now combining (4.9) with (4.11) leads to the following $L^{2}$-norm error estimate for the edge element scheme (4.2).

Lemma 4.2. Assume that $\operatorname{div}(\varepsilon \mathbf{E})=0$ and that $\mu^{-1} \in W^{1, \infty}(\Omega)$. Then the following estimate holds for $1 / 2<\delta^{\prime}<\delta_{\max }, \delta^{\prime} \leq 1$ :

$$
\left\|\mathbf{E}-\mathbf{E}_{h}\right\|_{0} \lesssim\left(h^{\delta^{\prime}}+\gamma(h)^{1 / 2}\right)\left\|\mathbf{E}-\mathbf{E}_{h}\right\|_{a_{h}}+\gamma(h)\|\mathbf{E}\|_{0}+\left\|\operatorname{div}\left(\varepsilon \mathbf{E}_{h}\right)\right\|_{-1} .
$$

Finally, the estimate for $\operatorname{div}\left(\varepsilon \mathbf{E}_{h}\right)$ follows directly from Lemma 3.8 since $\mathbf{E}_{h}$ is discrete $\varepsilon$-divergencefree. To see this, we suppose $\varepsilon \in W^{1, \infty}(\Omega)$ and $\left\{\mathcal{T}_{h}\right\}$ is quasi-uniform. Then for $1 / 2<s \leq 1$ and $1 / 2<\delta<\delta_{\max }^{D}, \delta \leq 1$, we have

$$
\left\|\operatorname{div}\left(\varepsilon \mathbf{E}_{h}\right)\right\|_{-s} \lesssim h^{s+\delta-1}\left\|\operatorname{curl} \mathbf{E}_{h}\right\|_{0} .
$$

Here $\left\|\mathbf{c u r l} \mathbf{E}_{h}\right\|_{0}$ can be further estimated as follows: From (4.2) and (4.3),

$$
\begin{aligned}
\left(\mu^{-1} \operatorname{curl} \mathbf{E}_{h}, \operatorname{curl} \mathbf{E}_{h}\right)+\gamma(h)\left(\varepsilon \mathbf{E}_{h}, \mathbf{E}_{h}\right) & =\left(\mu^{-1} \operatorname{curl} \mathbf{E}, \operatorname{curl} \mathbf{E}_{h}\right) \\
& \leq\|\operatorname{curl} \mathbf{E}\|_{0, \mu^{-1}}\left\|\operatorname{curl} \mathbf{E}_{h}\right\|_{0, \mu^{-1}},
\end{aligned}
$$

hence

$$
\left\|\operatorname{curl} \mathbf{E}_{h}\right\|_{0} \lesssim\left\|\operatorname{curl} \mathbf{E}_{h}\right\|_{0, \mu^{-1}} \leq\left\|\mathbf{E}_{h}\right\|_{a_{h}} \lesssim\|\operatorname{curl} \mathbf{E}\|_{0} .
$$

Summarizing the above results, we have the following theorem on the convergence of the edge element solution to (4.2), provided $\gamma(h)$ is suitably chosen.

Theorem 4.1. Suppose $\varepsilon \in W^{1, \infty}(\Omega), \mathbf{f} \in \mathbf{L}^{2}(\Omega)$, and $\left\{\mathcal{T}_{h}\right\}$ is quasi-uniform, and $\mathbf{E}$ and $\mathbf{E}_{h}$ are the solutions to the system (1.1)-(1.2) (with $\rho=0$ ) and the edge element scheme (4.2), respectively. Then it holds for $1 / 2<s \leq 1$ and $1 / 2<\delta<\delta_{\max }^{D}, \delta \leq 1$ that

$$
\left\|\operatorname{div}\left(\varepsilon \mathbf{E}_{h}\right)\right\|_{-s} \lesssim h^{s+\delta-1}\|\operatorname{curl} \mathbf{E}\|_{0} .
$$

If in addition we have $\mu^{-1} \in W^{1, \infty}(\Omega)$, and $\gamma(h)$ is a parameter such that $0<\gamma(h) \lesssim h^{2}$, then it holds for $1 / 2<\delta^{\prime}<\delta_{\max }, \delta^{\prime} \leq 1$ that

$$
\left\|\mathbf{E}-\mathbf{E}_{h}\right\|_{\mathbf{H}(\operatorname{curl} ; \Omega)} \lesssim h^{\delta^{\prime}}\|\mathbf{E}\|_{\mathbf{H}^{\delta^{\prime}}(\operatorname{curl} ; \Omega)} .
$$

Proof. (4.14) is a direct consequence of (4.12) and (4.13). On the other hand, from Lemma 3.6, we have $\mathbf{E} \in H^{\delta^{\prime}}(\operatorname{curl} ; \Omega)$. Then (4.15) follows from Lemmas 2.3, 4.1-4.2, (4.14) with $s=1$ and $\delta=\delta^{\prime}$, and the fact that $\gamma(h)^{1 / 2} \lesssim h$. The proof of the theorem is completed. 


\subsection{Error estimates for the case with charge}

In this section we establish the error estimates of the edge element scheme (4.4) for the case when charge is present, namely $\rho=\operatorname{div}(\varepsilon \mathbf{E}) \neq 0$. Most analyses are similar to those for the $\varepsilon$-divergence-free case in Section 4.3 , so we shall illustrate only those major differences.

We first estimate the error $\mathbf{e}:=\mathbf{E}-\mathbf{E}_{h}$ in the discrete energy norm. It follows from (4.3)-(4.4) that

$$
a_{h}\left(\mathbf{E}-\mathbf{E}_{h}, \mathbf{v}_{h}\right)=\gamma(h)\left(\varepsilon \mathbf{E}-\varepsilon \nabla \chi_{h}, \mathbf{v}_{h}\right), \quad \forall \mathbf{v}_{h} \in X_{h} .
$$

The following lemma can be proved in a similar manner to the proof of Lemma 4.1, along with the estimate that $\left\|\nabla \chi_{h}\right\|_{0, \varepsilon} \leq\|\nabla \chi\|_{0, \varepsilon} \lesssim\|\rho\|_{-1}$ (cf. (3.16)-(3.17)).

Lemma 4.3. Assume that $\rho \neq 0$, and $\gamma(h)$ is a parameter such that $0<\gamma(h) \lesssim 1$. Then the following estimate holds for the solutions $\mathbf{E}$ and $\mathbf{E}_{h}$ respectively to the system (1.1)-(1.2) and the edge element scheme (4.4):

$$
\left\|\mathbf{E}-\mathbf{E}_{h}\right\|_{a_{h}} \lesssim \inf _{\mathbf{v}_{h} \in X_{h}}\left\|\mathbf{E}-\mathbf{v}_{h}\right\|_{a_{h}}+\gamma(h)^{1 / 2}\left(\|\mathbf{E}\|_{0}+\|\rho\|_{-1}\right) .
$$

Next, we turn to the $L^{2}$-norm error estimate. As done in Section 4.3, it is easy to check that (4.9) remains the same while (4.10) becomes

$$
\begin{aligned}
\left\|\mathbf{e}_{0}\right\|_{0}^{2} & =a_{h}(\mathbf{e}, \mathbf{z})-\gamma(h)(\varepsilon \mathbf{e}, \mathbf{z}) \\
& =a_{h}\left(\mathbf{e}, \mathbf{z}-\mathbf{r}_{h} \mathbf{z}\right)+\gamma(h)\left(\varepsilon \mathbf{E}-\varepsilon \nabla \chi_{h}, \mathbf{r}_{h} \mathbf{z}\right)-\gamma(h)(\varepsilon \mathbf{e}, \mathbf{z}) .
\end{aligned}
$$

Then the estimate (4.11) in Section 4.3 changes now to

$$
\left\|\mathbf{e}_{0}\right\|_{0} \lesssim\left(h^{\delta^{\prime}}+\gamma(h)^{1 / 2}\right)\|\mathbf{e}\|_{a_{h}}+\gamma(h)\left(\|\mathbf{E}\|_{0}+\|\rho\|_{-1}\right)
$$

for $1 / 2<\delta^{\prime}<\delta_{\max }, \delta^{\prime} \leq 1$. Hence we obtain the following counterpart of Lemma 4.2.

Lemma 4.4. Suppose $\mu^{-1} \in W^{1, \infty}(\Omega)$ and $\left\{\mathcal{T}_{h}\right\}$ is quasi-uniform. Then it holds for $1 / 2<\delta^{\prime}<\delta_{\max }$, $\delta^{\prime} \leq 1$ that

$$
\left\|\mathbf{E}-\mathbf{E}_{h}\right\|_{0} \lesssim\left(h^{\delta^{\prime}}+\gamma(h)^{1 / 2}\right)\left\|\mathbf{E}-\mathbf{E}_{h}\right\|_{a_{h}}+\gamma(h)\left(\|\mathbf{E}\|_{0}+\|\rho\|_{-1}\right)+\left\|\operatorname{div}\left(\varepsilon \mathbf{E}-\varepsilon \mathbf{E}_{h}\right)\right\|_{-1} .
$$

It remains to estimate the error of the divergence $\operatorname{div}\left(\varepsilon \mathbf{E}_{h}\right)$. It is clear that

$$
\operatorname{div}(\varepsilon \mathbf{E})-\operatorname{div}\left(\varepsilon \mathbf{E}_{h}\right)=\rho-\operatorname{div} \varepsilon \mathbf{E}_{h}=\rho-\operatorname{div}\left(\varepsilon \nabla \chi_{h}\right)+\operatorname{div}\left(\varepsilon \nabla \chi_{h}\right)-\operatorname{div}\left(\varepsilon \mathbf{E}_{h}\right) .
$$

Since $\mathbf{E}_{h}-\nabla \chi_{h} \in X_{0, h}^{\varepsilon}$ is discrete $\varepsilon$-divergence-free (see Subsection 4.2), we can use Lemma 3.8. Suppose $\varepsilon \in W^{1, \infty}(\Omega)$ and $\left\{\mathcal{T}_{h}\right\}$ is quasi-uniform, then it holds for $1 / 2<s \leq 1$ and $1 / 2<\delta<\delta_{\text {max }}^{D}, \delta \leq 1$ that

$$
\left\|\operatorname{div}\left(\varepsilon \mathbf{E}_{h}\right)-\operatorname{div}\left(\varepsilon \nabla \chi_{h}\right)\right\|_{-s} \lesssim h^{s+\delta-1}\left\|\operatorname{curl} \mathbf{E}_{h}\right\|_{0}
$$

where we have used the fact that $\operatorname{curl} \nabla \chi_{h}=\mathbf{0}$. By combining (4.18), (4.19) and Lemma 3.9,

$$
\left\|\operatorname{div}(\varepsilon \mathbf{E})-\operatorname{div}\left(\varepsilon \mathbf{E}_{h}\right)\right\|_{-s} \lesssim h^{s+\delta-1}\left(\left\|\operatorname{curl} \mathbf{E}_{h}\right\|_{0}+\|\rho\|_{\delta-1}\right) .
$$

To further estimate $\left\|\operatorname{curl} \mathbf{E}_{h}\right\|_{0}$, we obtain from (4.4) and (4.3) that

$$
\begin{aligned}
\left(\mu^{-1} \operatorname{curl} \mathbf{E}_{h}, \operatorname{curl} \mathbf{E}_{h}\right)+\gamma(h)\left(\varepsilon \mathbf{E}_{h}, \mathbf{E}_{h}\right) & =\left(\mu^{-1} \operatorname{curl} \mathbf{E}, \operatorname{curl} \mathbf{E}_{h}\right)+\gamma(h)\left(\varepsilon \nabla \chi_{h}, \mathbf{E}_{h}\right) \\
& \leq\left(\|\operatorname{curl} \mathbf{E}\|_{0, \mu^{-1}}+\gamma(h)^{1 / 2}\left\|\nabla \chi_{h}\right\|_{0, \varepsilon}\right)\left\|\mathbf{E}_{h}\right\|_{a_{h}},
\end{aligned}
$$

which implies

$$
\left\|\operatorname{curl} \mathbf{E}_{h}\right\|_{0} \lesssim\left\|\mathbf{E}_{h}\right\|_{a_{h}} \lesssim\|\operatorname{curl} \mathbf{E}\|_{0}+\gamma(h)^{1 / 2}\|\rho\|_{-1} .
$$

Now following the proof of Theorem 4.1 we come to the conclusion of the next theorem by using Lemmas 4.3-4.4, the estimates (4.20)-(4.21), and the fact that $\|\rho\|_{\delta^{\prime}-1}=\|\operatorname{div}(\varepsilon \mathbf{E})\|_{\delta^{\prime}-1} \lesssim\|\mathbf{E}\|_{\delta^{\prime}}$. 
Theorem 4.2. Suppose $\varepsilon \in W^{1, \infty}(\Omega), \mathbf{f} \in \mathbf{L}^{2}(\Omega), \rho \in L^{2}(\Omega)$ and $\left\{\mathcal{T}_{h}\right\}$ is quasi-uniform. Let $\mathbf{E}$ and $\mathbf{E}_{h}$ be the solutions to the system (1.1)-(1.2), and the edge element scheme (4.4) respectively, then it holds for $1 / 2<s \leq 1$ and $1 / 2<\delta<\delta_{\max }^{D}, \delta \leq 1$ that

$$
\left\|\operatorname{div}(\varepsilon \mathbf{E})-\operatorname{div}\left(\varepsilon \mathbf{E}_{h}\right)\right\|_{-s} \lesssim h^{s+\delta-1}\left(\|\operatorname{curl} \mathbf{E}\|_{0}+\|\rho\|_{\delta-1}\right) .
$$

If in addition we have $\mu^{-1} \in W^{1, \infty}(\Omega)$, and choose $\gamma(h)$ such that $0<\gamma(h) \lesssim h^{2}$, then the following error estimates are satisfied for $1 / 2<\delta^{\prime}<\delta_{\max }, \delta^{\prime} \leq 1$ :

$$
\left\|\mathbf{E}-\mathbf{E}_{h}\right\|_{\mathbf{H}(\operatorname{curl} ; \Omega)} \lesssim h^{\delta^{\prime}}\|\mathbf{E}\|_{\mathbf{H}^{\delta^{\prime}}(\operatorname{curl} ; \Omega)} .
$$

Remark 4.3. Two important remarks about the major results of this paper are in order.

(a) All the error estimates in Theorems 4.1 and 4.2, as well as in the subsequent Theorems 5.1 and 7.1 , are uniform with respect to the parameter $\gamma(h)$ in the range $0<\gamma(h) \lesssim h^{2}$.

(b) The analysis of this paper focuses only on the lowest order edge elements. It is interesting to see if the analysis can be generalized to higher order edge elements, which are expected for solving problems in smooth domains with smooth data. For such a generalization, it is natural to require parameter $\gamma(h)$ to be selected in the range $0<\gamma(h) \lesssim h^{\alpha}$ with $\alpha>2$. But a major technical issue for such a generalization relies on if a discrete $\varepsilon$-divergence-free function can be approximated by a continuous $\varepsilon$-divergence-free function with a desired higher order accuracy (cf. Lemma 3.7).

\subsection{Relation with the standard saddle-point system}

As it was mentioned at the beginning of Section 4, one usually solves the stationary problem (1.1)-(1.2) with the help of the saddle-point system: Find $\left(\mathbf{E}_{h}^{\dagger}, p_{h}^{\dagger}\right) \in X_{h} \times U_{h}$ such that

$$
\begin{aligned}
& \left(\mu^{-1} \operatorname{curl} \mathbf{E}_{h}^{\dagger}, \operatorname{curl} \mathbf{v}_{h}\right)+\left(\varepsilon \mathbf{v}_{h}, \nabla p_{h}^{\dagger}\right)=\left(\mathbf{f}, \mathbf{v}_{h}\right), \quad \forall \mathbf{v}_{h} \in X_{h} \\
& \left(\varepsilon \mathbf{E}_{h}^{\dagger}, \nabla q_{h}\right)=-\left(\rho, q_{h}\right), \quad \forall q_{h} \in U_{h},
\end{aligned}
$$

where the divergence law (1.2) is enforced explicitly through the second variational equation above. In this subsection we shall discuss some interesting relationship between the solution $\mathbf{E}_{h}^{\dagger}$ to the standard saddle-point system (4.24) and the solution $\mathbf{E}_{h}$ to the newly proposed edge element scheme (4.4).

The saddle-point system (4.24) is well-posed, and using the Babuska-Brezzi theory [20, 9], one finds that the convergence of its solution in $\mathbf{H}(\mathbf{c u r l})$-norm is the same as (4.23) (cf. [15]). In other words, under the same assumptions as those in Theorem 4.2, it holds for $1 / 2<\delta^{\prime}<\delta_{\max }$ and $\delta^{\prime} \leq 1$ that

$$
\left\|\mathbf{E}-\mathbf{E}_{h}^{\dagger}\right\|_{\mathbf{H}(\operatorname{curl} ; \Omega)} \lesssim h^{\delta^{\prime}}\|\mathbf{E}\|_{\mathbf{H}^{\delta^{\prime}}(\operatorname{curl} ; \Omega)} .
$$

Also, we can see that $\nabla p_{h}^{\dagger}=0$ by taking $\mathbf{v}_{h}=\nabla p_{h}^{\dagger}$ in the first equation of (4.24) and then integrating by parts, hence the Lagrange multiplier $p_{h}^{\dagger}$ in (4.24) is actually identical to zero.

Clearly, convergence of $\mathbf{E}_{h}-\mathbf{E}_{h}^{\dagger}$ to zero in $\mathbf{H}$ (curl)-norm is an obvious consequence of (4.23) and (4.25). Next we study the convergence of $\mathbf{E}_{h}-\mathbf{E}_{h}^{\dagger}$ in the divergence form. By the definitions of $\mathbf{E}_{h}$ and $\mathbf{E}_{h}^{\dagger}$, we can easily check that

$$
\left(\mu^{-1} \operatorname{curl}\left(\mathbf{E}_{h}-\mathbf{E}_{h}^{\dagger}\right), \operatorname{curl} \mathbf{v}_{h}\right)=\gamma(h)\left(\varepsilon\left(\nabla \chi_{h}-\mathbf{E}_{h}\right), \mathbf{v}_{h}\right), \quad \forall \mathbf{v}_{h} \in X_{h},
$$

where $\chi_{h} \in U_{h}$ is the solution to (3.16). We know from (3.16), (4.5) and (4.24) that the difference $\mathbf{E}_{h}-\mathbf{E}_{h}^{\dagger}$ is discrete $\varepsilon$-divergence-free, namely $\mathbf{E}_{h}-\mathbf{E}_{h}^{\dagger} \in X_{0, h}^{\varepsilon}$. Using this fact and taking $\mathbf{v}_{h}=\mathbf{E}_{h}-\mathbf{E}_{h}^{\dagger}$ in (4.26), we obtain

$$
\left\|\mu^{-1 / 2} \operatorname{curl}\left(\mathbf{E}_{h}-\mathbf{E}_{h}^{\dagger}\right)\right\|^{2}=-\gamma(h)\left(\varepsilon \mathbf{E}_{h}, \mathbf{E}_{h}-\mathbf{E}_{h}^{\dagger}\right) .
$$

But we know readily from (4.23) that $\left\|\mathbf{E}_{h}\right\|_{0} \lesssim\|\mathbf{E}\|_{\left.\mathbf{H}^{\delta^{\prime}} \text { (curl; } ; \Omega\right)}$, and from (4.23) and (4.25) that

$$
\left\|\mathbf{E}_{h}-\mathbf{E}_{h}^{\dagger}\right\|_{0} \leq\left\|\mathbf{E}_{h}-\mathbf{E}\right\|_{0}+\left\|\mathbf{E}-\mathbf{E}_{h}^{\dagger}\right\|_{0} \lesssim h^{\delta^{\prime}}\|\mathbf{E}\|_{\mathbf{H}^{\delta^{\prime}}(\operatorname{curl} ; \Omega)} .
$$


Combining these two estimates with (4.27) gives

$$
\left\|\mu^{-1 / 2} \operatorname{curl}\left(\mathbf{E}_{h}-\mathbf{E}_{h}^{\dagger}\right)\right\|_{0} \lesssim h^{\delta^{\prime} / 2}(\gamma(h))^{1 / 2}\|\mathbf{E}\|_{\mathbf{H}^{\delta^{\prime}}(\operatorname{curl} ; \Omega)}
$$

for $1 / 2<\delta^{\prime}<\delta_{\max }$ and $\delta^{\prime} \leq 1$. On the other hand, it follows from (4.28) and Lemma 3.8 that for $1 / 2<\delta<\delta_{\max }^{D}, \delta \leq 1$ and $1 / 2<s \leq 1$,

$$
\left\|\operatorname{div}\left(\varepsilon \mathbf{E}_{h}\right)-\operatorname{div}\left(\varepsilon \mathbf{E}_{h}^{\dagger}\right)\right\|_{-s} \lesssim h^{s+\delta+\delta^{\prime} / 2-1}(\gamma(h))^{1 / 2}\|\mathbf{E}\|_{\mathbf{H}^{\delta^{\prime}}(\mathbf{c u r l} ; \Omega)} .
$$

This proves the convergence of $\mathbf{E}_{h}-\mathbf{E}_{h}^{\dagger}$ to zero in both $\mathbf{H}(\mathbf{c u r l})$-norm and the $\varepsilon$-divergence form. As a by-product, the combination of the last estimate above with (4.22) leads to an error estimate of the divergence of the edge element solution $\mathbf{E}_{h}^{\dagger}$ to the standard saddle-point system (4.24).

Finally, we consider the convergence of $\mathbf{E}_{h}$ to $\mathbf{E}_{h}^{\dagger}$ when the mesh size $h$ is fixed but $\gamma:=\gamma(h)$ tends to zero. We first know from (4.28) and (4.29) that both $\left\|\mathbf{c u r l} \mathbf{E}_{h}-\mathbf{c u r l} \mathbf{\mathbf { E } _ { h } ^ { \dagger }}\right\|_{0}$ and $\left\|\operatorname{div}\left(\varepsilon \mathbf{E}_{h}\right)-\operatorname{div}\left(\varepsilon \mathbf{E}_{h}^{\dagger}\right)\right\|$ converges to zero in the rate $\gamma^{1 / 2}$. We end this section with the convergence of the $L^{2}$-norm $\left\|\mathbf{E}_{h}-\mathbf{E}_{h}^{\dagger}\right\|_{0}$, by using a continuous embedding result that generalizes the one of Corollary 3.1. To do so, we introduce

$$
\mathbf{X}_{N,-s}(\Omega, \varepsilon):=\left\{\mathbf{u} \in \mathbf{H}_{0}(\operatorname{curl} ; \Omega) ; \operatorname{div}(\varepsilon \mathbf{u}) \in H^{-s}(\Omega)\right\}
$$

for $1 / 2<s<1$. By the definition of the sedge elements, one has $\mathbf{E}_{h}-\mathbf{E}_{h}^{\dagger} \in \mathbf{X}_{N,-s}(\Omega, \varepsilon)$ for all $1 / 2<s<1$. Adapting the proof of Lemma 3.1 in [7], one finds that

Corollary 4.1. Suppose $\varepsilon \in W^{1, \infty}(\Omega)$. For $1 / 2<s<1$, it holds that

$$
\mathbf{X}_{N,-s}(\Omega, \varepsilon) \subset \bigcap_{0 \leq \delta \leq 1-s} H^{\delta}(\Omega) .
$$

In particular, $\mathbf{X}_{N,-s}(\Omega, \varepsilon)$ are compact subsets of $\mathbf{L}^{2}(\Omega)$, and we can show the following result in the same argument as in the proof of Corollary 3.2 .

Corollary 4.2. For $1 / 2<s<1$, the seminorm

$$
\mathbf{u} \mapsto\left(\|\operatorname{curl} \mathbf{u}\|_{0}^{2}+\|\operatorname{div}(\varepsilon \mathbf{u})\|_{-s}^{2}\right)^{1 / 2}
$$

is a norm of $\mathbf{X}_{N,-s}(\Omega, \varepsilon)$, which is equivalent to its full norm.

Using this Corollary and (4.28)-(4.29), we know that $\left\|\mathbf{E}_{h}-\mathbf{E}_{h}^{\dagger}\right\|_{0}$ converges to zero also in the rate $\gamma^{1 / 2}$ when $h$ is fixed but $\gamma$ tends to zero.

\section{Stationary problem with discontinuous coefficients}

In this section we extend the convergence analyses in Sections 4.3-4.4 on the edge element approximations (4.2) and (4.4) for the stationary problem (1.1)-(1.2) to the case with discontinuous coefficients $\varepsilon$ and $\mu$.

We assume that the triangulations $\left\{\mathcal{T}_{h}\right\}$ are consistent with respect to the partition $\mathcal{P}=\left\{\Omega_{j}\right\}_{j=1}^{J}$ of $\Omega$ based on the distribution of coefficients $\varepsilon$ and $\mu$ (see Section 3.1), namely, for each $K \in \mathcal{T}_{h}$ there exists a single index $j$ such that $\operatorname{int}(K) \cap \Omega_{j} \neq \emptyset$. Then for functions in the space $\mathbf{H}(\mathbf{c u r l} ; \Omega) \cap \mathbf{P H} \mathbf{H}^{r}(\mathbf{c u r l} ; \Omega)$, we have similar interpolation error estimates to the ones in Lemma 2.3 by extending the analyses in [16] or [29, Theorem 5.41].

Lemma 5.1. For any $\mathbf{u} \in \mathbf{H}(\operatorname{curl} ; \Omega) \cap \mathbf{P} \mathbf{H}^{r}(\operatorname{curl} ; \Omega)$ with $1 / 2<r \leq 1$ it holds that

$$
\left\|\mathbf{u}-\mathbf{r}_{h} \mathbf{u}\right\|_{\mathbf{H}(\operatorname{curl} ; \Omega)} \lesssim h^{r}\|\mathbf{u}\|_{\mathbf{P H}(\operatorname{curl} ; \Omega)} .
$$


It is crucial for us to observe from Section 3.1 that for a geometry of type (G1) or (G2), the $\varepsilon$ divergence of functions in $\mathbf{X}_{N}(\Omega, \varepsilon)$ is "sufficiently regular" to suit our convergence analysis. Indeed, for a $\mathbf{u} \in \mathbf{X}_{N}(\Omega, \varepsilon)$, we know $\varepsilon \mathbf{u} \in \mathbf{H}^{\eta}(\Omega)$ for all $\eta \in[0,1 / 2)$, hence $\operatorname{div}(\varepsilon \mathbf{u})$ belongs to $H^{-s}(\Omega)$ for $1 / 2<s \leq 1$.

Now by simply replacing the appropriate norms by their broken counterparts for piecewise smooth functions, we can basically follow the analyses in Section 4, especially the proof of Theorem 4.2, to derive the error estimates of the solution to the edge element scheme (4.4) for the stationary problem (1.1)-(1.2) with discontinuous coefficients.

Theorem 5.1. Suppose $\varepsilon$ and $\mu$ are piecewise constant over the domain $\Omega$ with geometry of type (G1) or (G2), $\mathbf{f} \in \mathbf{L}^{2}(\Omega), \rho \in L^{2}(\Omega)$ and $\left\{\mathcal{T}_{h}\right\}$ is quasi-uniform. Let $\mathbf{E}$ and $\mathbf{E}_{h}$ be the solutions to the system (1.1)-(1.2) and the edge element scheme (4.4) respectively. Then the following error estimate holds for $1 / 2<s \leq 1$ and $1 / 2<\delta<\delta_{\max }^{D}, \delta \leq 1$ that

$$
\left\|\operatorname{div}(\varepsilon \mathbf{E})-\operatorname{div}\left(\varepsilon \mathbf{E}_{h}\right)\right\|_{-s} \lesssim h^{s+\delta-1}\left(\|\operatorname{curl} \mathbf{E}\|_{0}+\|\rho\|_{\delta-1}\right) .
$$

If $\gamma(h)$ is chosen such that $0<\gamma(h) \lesssim h^{2}$, then it holds for $1 / 2<\delta^{\prime}<\delta_{\max }, \delta^{\prime} \leq 1$ that

$$
\left\|\mathbf{E}-\mathbf{E}_{h}\right\|_{\mathbf{H}(\operatorname{curl} ; \Omega)} \lesssim h^{\delta^{\prime}}\|\mathbf{E}\|_{\mathbf{P H}^{\delta^{\prime}}(\operatorname{curl} ; \Omega)} .
$$

Remark 5.1. The same convergence orders still hold true as in (5.1) and (5.2) under the conditions $\chi \in P H^{1+\delta}(\Omega)$ and $\mathbf{E} \in \mathbf{P H}^{\delta^{\prime}}(\mathbf{c u r l} ; \Omega)$, where $\chi$ is the solution to (3.17) (see the proof of Lemma 3.9). In other words, one may use these conditions on $\chi$ and $\mathbf{E}$ to replace the assumption on the geometry of the domain and its partition.

\section{Time-harmonic Maxwell system}

In this section we shall extend the convergence results in the previous Sections 4-5 for the stationary system (1.1)-(1.2) to the time-harmonic Maxwell system (1.3)-(1.4). All the notations below will be kept to be the same as in the previous sections. Then we can readily have the following weak formulation for (1.3)-(1.4) and its edge element approximation:

Find $\mathbf{E} \in \mathbf{H}_{0}(\mathbf{c u r l} ; \Omega)$ such that

$$
\left(\mu^{-1} \operatorname{curl} \mathbf{E}, \operatorname{curl} \mathbf{v}\right)-k^{2}(\varepsilon \mathbf{E}, \mathbf{v})=(\mathbf{f}, \mathbf{v}) \quad \forall \mathbf{v} \in \mathbf{H}_{0}(\operatorname{curl} ; \Omega) ;
$$

Find $\mathbf{E}_{h} \in X_{h}$ such that

$$
\left(\mu^{-1} \operatorname{curl} \mathbf{E}_{h}, \operatorname{curl} \mathbf{v}_{h}\right)-k^{2}\left(\varepsilon \mathbf{E}_{h}, \mathbf{v}_{h}\right)=\left(\mathbf{f}, \mathbf{v}_{h}\right) \quad \forall \mathbf{v}_{h} \in X_{h} .
$$

The first estimate below in $\mathbf{H}$ (curl )-norm is standard (cf. [29, Theorem 7.1] [12]). Then following the convergence analysis in Sections 4-5, we can further achieve more specific error estimates as stated in the following lemma.

Lemma 6.1. There is a threshold value $h_{0}>0$ such that it holds for $0<h \leq h_{0}$ that

$$
\left\|\mathbf{E}-\mathbf{E}_{h}\right\|_{\mathbf{H}(\operatorname{curl} ; \Omega)} \lesssim \inf _{\mathbf{v}_{h} \in X_{h}}\left\|\mathbf{E}-\mathbf{v}_{h}\right\|_{\mathbf{H}(\operatorname{curl} ; \Omega)} .
$$

Suppose that $\mathbf{f} \in \mathbf{L}^{2}(\Omega), \rho \in L^{2}(\Omega)$, and

- either $\varepsilon, \mu^{-1} \in W^{1, \infty}(\Omega)$;

- or $\varepsilon, \mu$ are piecewise constant over the domain $\Omega$ with geometry of type (G1) or (G2).

Then one has the following error estimate for $1 / 2<\delta^{\prime}<\delta_{\max }, \delta^{\prime} \leq 1$ :

$$
\left\|\mathbf{E}-\mathbf{E}_{h}\right\|_{\mathbf{H}(\mathbf{c u r l} ; \Omega)} \lesssim h^{\delta^{\prime}}\|\mathbf{E}\|_{\mathbf{P H}^{\delta^{\prime}}(\mathbf{c u r l} ; \Omega)} .
$$

Next we establish the error estimate for the divergence of $\varepsilon \mathbf{E}_{h}$. 
Theorem 6.1. Suppose $\left\{\mathcal{T}_{h}\right\}$ is quasi-uniform. Then under the same assumptions as in Lemma 6.1, it holds for $0<h \leq h_{0}, 1 / 2<s \leq 1$ and $1 / 2<\delta<\delta_{\max }^{D}, \delta \leq 1$ that

$$
\left\|\operatorname{div}(\varepsilon \mathbf{E})-\operatorname{div}\left(\varepsilon \mathbf{E}_{h}\right)\right\|_{-s} \lesssim h^{s+\delta-1}\left(\|\operatorname{curl} \mathbf{E}\|_{0}+\|\rho\|_{\delta-1}\right) .
$$

Proof. For any $\varphi_{h} \in U_{h}$ it follows from (6.1)-(6.2) that

$$
\left(\varepsilon \mathbf{E}, \nabla \varphi_{h}\right)=\left(\varepsilon \mathbf{E}_{h}, \nabla \varphi_{h}\right),
$$

that is, $\mathbf{E}-\mathbf{E}_{h}$ is discrete $\varepsilon$-divergence-free. On the other hand, we know from (1.4), the relation (6.3) and the definition of $\chi_{h}$ in (3.16) that $\mathbf{E}-\nabla \chi_{h}$ is also discrete $\varepsilon$-divergence-free, so is $\nabla \chi_{h}-\mathbf{E}_{h}$. Noting that $\nabla \chi_{h}-\mathbf{E}_{h} \in X_{0, h}^{\varepsilon}$ and $\operatorname{curl} \nabla \chi_{h}=\mathbf{0}$, we obtain from Lemma 3.8 that for $1 / 2<s \leq 1$,

$$
\left\|\operatorname{div}\left(\varepsilon \nabla \chi_{h}\right)-\operatorname{div}\left(\varepsilon \mathbf{E}_{h}\right)\right\|_{-s} \lesssim h^{s+\delta-1}\left\|\operatorname{curl}\left(\nabla \chi_{h}-\mathbf{E}_{h}\right)\right\|_{0}=h^{s+\delta-1}\left\|\operatorname{curl} \mathbf{E}_{h}\right\|_{0} .
$$

Furthermore, we can derive from Lemma 3.9 that

$$
\left\|\operatorname{div}(\varepsilon \mathbf{E})-\operatorname{div}\left(\varepsilon \nabla \chi_{h}\right)\right\|_{-s} \lesssim h^{s+\delta-1}\|\rho\|_{\delta-1} .
$$

The above two estimates imply

$$
\left\|\operatorname{div}(\varepsilon \mathbf{E})-\operatorname{div}\left(\varepsilon \mathbf{E}_{h}\right)\right\|_{-s} \lesssim h^{s+\delta-1}\left(\left\|\operatorname{curl} \mathbf{E}_{h}\right\|_{0}+\|\rho\|_{\delta-1}\right) .
$$

Now the desired estimate follows from the estimate $\left\|\mathbf{c u r l} \mathbf{E}_{h}\right\|_{0} \lesssim\|\mathbf{E}\|_{\mathbf{H}(\mathbf{c u r l} ; \Omega)}$, which is a direct consequence of Lemma 6.1 with $\mathbf{v}_{h}=0$ for $0<h \leq h_{0}$.

\section{Time-dependent Maxwell system}

In this section we consider a fully discrete edge element approximation to the time-dependent Maxwell system (1.5)-(1.7) and its error estimates. Let us first introduce our fully discrete edge element approximation. To do so, we divide the time interval $(0, T)$ into $M$ equally spaced subintervals using the nodal points $0=t^{0}<t^{1}<\cdots<t^{M}=T$, with $t^{n}=n \tau$ and $\tau=T / M$. From now on, for any function $u(t, \mathbf{x})$ we may write $u\left(t^{n}, \mathbf{x}\right)$ as $u^{n}$. Then for a given sequence $\left\{u^{n}\right\}_{n=0}^{M}$ in $L^{2}(\Omega)$ or $\mathbf{L}^{2}(\Omega)$, we define its first and second order backward differences by

$$
\partial_{\tau} u^{n}=\frac{u^{n}-u^{n-1}}{\tau}, \quad \partial_{\tau}^{2} u^{n}=\frac{\partial_{\tau} u^{n}-\partial_{\tau} u^{n-1}}{\tau}
$$

As in [16], we consider the following edge element approximation of the time-dependent problem (1.5)(1.7): Find $\mathbf{E}_{h}^{m} \in X_{h}$ for $m=1,2, \cdots, M$ such that

$$
\left(\varepsilon \partial_{\tau}^{2} \mathbf{E}_{h}^{m}, \mathbf{v}_{h}\right)+\left(\mu^{-1} \operatorname{curl} \mathbf{E}_{h}^{m}, \operatorname{curl} \mathbf{v}_{h}\right)=\left(\mathbf{f}^{m}, \mathbf{v}_{h}\right), \quad \forall \mathbf{v}_{h} \in X_{h} .
$$

We recall that the initial values $\mathbf{E}_{h}^{0} \in X_{h}$ and $\mathbf{E}_{h}^{-1} \in X_{h}$ are usually given by the natural edge element interpolations of the exact initial values $\mathbf{E}^{0}$ and $\mathbf{F}^{0}$ in most existing methods. But these approximations will not ensure a strong convergence of the divergence law in appropriate norm, as we did in the previous sections for the stationary system (1.1)-(1.2) and the time-harmonic system (1.3)-(1.4). Instead, we define the initial value $\mathbf{E}_{h}^{0} \in X_{h}$ by

$$
a_{h}\left(\mathbf{E}_{h}^{0}, \mathbf{v}_{h}\right)=\left(\mu^{-1} \operatorname{curl} \mathbf{E}^{0}, \operatorname{curl} \mathbf{v}_{h}\right)+\gamma(h)\left(\varepsilon \nabla \chi_{h}^{0}, \mathbf{v}_{h}\right), \quad \forall \mathbf{v}_{h} \in X_{h}
$$

and the initial value $\mathbf{E}_{h}^{-1} \in X_{h}$ by $\mathbf{E}_{h}^{0}-\mathbf{E}_{h}^{-1}=\tau \mathbf{F}_{h}^{0}$, where $\mathbf{F}_{h}^{0} \in X_{h}$ solves the discrete system:

$$
a_{h}\left(\mathbf{F}_{h}^{0}, \mathbf{v}_{h}\right)=\left(\mu^{-1} \operatorname{curl} \mathbf{F}^{0}, \operatorname{curl} \mathbf{v}_{h}\right)+\gamma(h)\left(\varepsilon \nabla \theta_{h}^{0}, \mathbf{v}_{h}\right), \quad \forall \mathbf{v}_{h} \in X_{h} .
$$

Here the bilinear form $a_{h}$ is defined by (4.1) and $\chi_{h}^{0}, \theta_{h}^{0} \in U_{h}$ are defined respectively by

$$
\left(\varepsilon \nabla \chi_{h}^{0}, \nabla \varphi_{h}\right)=-\left(\rho^{0}, \varphi_{h}\right), \quad\left(\varepsilon \nabla \theta_{h}^{0}, \nabla \varphi_{h}\right)=-\left(\rho_{t}^{0}, \varphi_{h}\right), \quad \forall \varphi_{h} \in U_{h} .
$$


Remark 7.1. Instead of the first order backward difference in time used in the fully discrete scheme (7.1)(7.4), one can also use some second order difference approximations in time, e.g., the Crank-Nicolson scheme [16].

We first present an analogue of Lemma 3.8, providing an estimate of the divergence of an edge element function in $H^{-s}(\Omega)$-norm for $1 / 2<s \leq 1$.

Lemma 7.1. Suppose $\varepsilon$ and $\mu$ satisfy the same assumptions as in Lemma 6.1 and $\left\{\mathcal{T}_{h}\right\}$ is quasi-uniform. Assume that $\mathbf{w}_{h} \in X_{h}$ satisfies

$$
\left(\varepsilon \mathbf{w}_{h}, \nabla \varphi_{h}\right)=-\left\langle r, \varphi_{h}\right\rangle, \quad \forall \varphi_{h} \in U_{h},
$$

where $r \in H^{-s}(\Omega)$ for some $1 / 2<s \leq 1$ and $\langle\cdot, \cdot\rangle$ denotes the dual pairing between $H^{-s}(\Omega)$ and $H_{0}^{s}(\Omega)$. Then for $1 / 2<\delta<\delta_{\max }^{D}, \delta \leq 1$ :

$$
\left\|\operatorname{div}\left(\varepsilon \mathbf{w}_{h}\right)\right\|_{-s} \lesssim h^{s+\delta-1}\left\|\operatorname{curl}_{h}\right\|_{0}+\|r\|_{-s} .
$$

Proof. By following the proof of Lemma 3.8 we may show that

$$
\begin{aligned}
\left\|\operatorname{div}\left(\varepsilon \mathbf{w}_{h}\right)\right\|_{-s} \lesssim h^{s+\delta-1}\left\|\operatorname{curl}_{h}\right\|_{0}+\sup _{0 \neq \varphi \in H_{0}^{s}(\Omega)} \frac{\left|\left\langle\varepsilon \mathbf{w}_{h}, \nabla \Pi_{h} \varphi\right\rangle\right|}{\|\varphi\|_{s}} \\
\lesssim h^{s+\delta-1}\left\|\operatorname{curl}_{h}\right\|_{0}+\sup _{0 \neq \varphi \in H_{0}^{s}(\Omega)} \frac{\left|\left\langle r, \Pi_{h} \varphi\right\rangle\right|}{\|\varphi\|_{s}} .
\end{aligned}
$$

Then the desired estimate follows by noting that $\left|\left\langle r, \Pi_{h} \varphi\right\rangle\right| \leq\|r\|_{-s}\left\|\Pi_{h} \varphi\right\|_{s} \lesssim\|r\|_{-s}\|\varphi\|_{s}$. This completes the proof of the lemma.

Now we are ready to establish the error estimates for the fully discrete scheme (7.1)-(7.3).

Theorem 7.1. Suppose $\left\{\mathcal{T}_{h}\right\}$ is quasi-uniform. Let $\mathbf{E}$ and $\mathbf{E}_{h}$ be the solutions to the Maxwell system (1.5)-(1.7) and its edge element scheme (7.1)-(7.4) respectively, where $\gamma(h)$ is chosen such that $0<$ $\gamma(h) \lesssim h^{2}$, and let $\chi$ be the solution to (3.17). Assume the following regularities hold for $\mathbf{E}$ and $\chi$ for some $1 / 2<\delta \leq 1$ :

$$
\left\{\begin{array}{l}
\mathbf{E} \in H^{2}\left(0, T ; \mathbf{H}_{0}(\mathbf{c u r l} ; \Omega) \cap \mathbf{P H}^{\delta}(\operatorname{curl} ; \Omega)\right) \cap H^{3}\left(0, T ; \mathbf{L}^{2}(\Omega)\right) \\
\chi \in L^{2}\left(0, T ; \mathrm{PH}^{1+\delta}(\Omega)\right), \chi^{0}, \chi_{t}^{0} \in \mathrm{PH}^{1+\delta}(\Omega) ; \\
\rho \in W^{3,1}\left(0, T ; H^{-1}(\Omega)\right) \cap L^{\infty}\left(0, T ; L^{2}(\Omega)\right), \rho^{0}, \rho_{t}^{0} \in L^{2}(\Omega) .
\end{array}\right.
$$

Then we have the following error estimates

$$
\max _{1 \leq m \leq M}\left(\left\|\partial_{\tau} \mathbf{E}_{h}^{m}-\mathbf{E}_{t}^{m}\right\|_{0}^{2}+\left\|\operatorname{curl}\left(\mathbf{E}_{h}^{m}-\mathbf{E}^{m}\right)\right\|_{0}^{2}\right) \lesssim\left(\tau^{2}+\tau^{2} h^{2(\delta-1)}+h^{2 \delta}\right) .
$$

In addition, the following error estimates hold for $1 / 2<s \leq 1$ and $0 \leq m \leq M$ that

$$
\begin{aligned}
& \left\|\operatorname{div}\left(\varepsilon \mathbf{E}^{m}\right)-\operatorname{div}\left(\varepsilon \mathbf{E}_{h}^{m}\right)\right\|_{-s} \lesssim \tau\left(\left\|\rho_{t t}^{0}\right\|_{-s}+\int_{0}^{t^{m}}\left\|\rho_{t t t}\right\|_{-s}\right) \\
& +h^{s+\delta-1}\left(\left\|\mathbf{F}^{0}\right\|_{\mathbf{H}(\mathbf{c u r l} ; \Omega)}+\left\|\operatorname{curl} \mathbf{E}^{0}\right\|_{0}+\left\|\rho^{0}\right\|_{-1}+\left\|\rho_{t}^{0}\right\|_{-1}+\left\|\rho^{m}\right\|_{\delta-1}+\left(\sum_{j=1}^{m} \tau\left\|\mathbf{f}^{j}\right\|_{0}^{2}\right)^{1 / 2}\right) .
\end{aligned}
$$

Proof. Note that $\operatorname{div}\left(\varepsilon \mathbf{E}^{0}\right)=\rho^{0}$ and $\operatorname{div}\left(\varepsilon \mathbf{F}^{0}\right)=\rho_{t}^{0}$. So we may directly apply Theorem 4.2 or 5.1 respectively to the edge element scheme (7.2) for approximating $\mathbf{E}^{0}$ and the edge element scheme (7.3) for approximating $\mathbf{F}^{0}$ to obtain

$$
\begin{aligned}
\left\|\mathbf{E}^{0}-\mathbf{E}_{h}^{0}\right\|_{\mathbf{H}(\operatorname{curl} ; \Omega)} & \lesssim h^{\delta}\left(\left\|\mathbf{E}^{0}\right\|_{\mathbf{P H}^{\delta}(\operatorname{curl} ; \Omega)}+\left\|\chi^{0}\right\|_{\mathrm{PH}^{1+\delta}(\Omega)}\right), \\
\left\|\mathbf{F}^{0}-\mathbf{F}_{h}^{0}\right\|_{\mathbf{H}(\operatorname{curl} ; \Omega)} & \lesssim h^{\delta}\left(\left\|\mathbf{E}_{t}^{0}\right\|_{\mathbf{P H}^{\delta}(\operatorname{curl} ; \Omega)}+\left\|\chi_{t}^{0}\right\|_{\mathrm{PH}^{1+\delta}(\Omega)}\right) .
\end{aligned}
$$


Moreover, we have the following error estimates for the divergence for $1 / 2<s \leq 1$ :

$$
\begin{aligned}
&\left\|\operatorname{div}\left(\varepsilon \mathbf{E}^{0}\right)-\operatorname{div}\left(\varepsilon \mathbf{E}_{h}^{0}\right)\right\|_{-s} \lesssim h^{s+\delta-1}\left(\left\|\operatorname{curl} \mathbf{E}^{0}\right\|_{0}+\left\|\rho^{0}\right\|_{\delta-1}\right), \\
&\left\|\operatorname{div}\left(\varepsilon \mathbf{F}^{0}\right)-\operatorname{div}\left(\varepsilon \mathbf{F}_{h}^{0}\right)\right\|_{-s} \lesssim h^{s+\delta-1}\left(\left\|\operatorname{curl} \mathbf{F}^{0}\right\|_{0}+\left\|\rho_{t}^{0}\right\|_{\delta-1}\right) .
\end{aligned}
$$

Now the $\mathbf{H}$ (curl ) error estimate (7.5) can be derived by following the arguments in [16] using (7.7). It remains to establish the error estimate (7.6). For this we define $\chi_{h}^{m} \in U_{h}$ for $m=0,1,2, \cdots, M$ by

$$
\left(\varepsilon \nabla \chi_{h}^{m}, \nabla \varphi_{h}\right)=-\left(\rho^{m}, \varphi_{h}\right), \quad \forall \varphi_{h} \in U_{h},
$$

and define $\chi_{h}^{-1}:=\chi_{h}^{0}-\tau \theta_{h}^{0}$, where $\theta_{h}^{0}$ is given by (7.4). We shall write $\rho^{-1}:=\rho^{0}-\tau \rho_{t}^{0}$.

Using the fact that $\operatorname{div} \mathbf{f}=\rho_{t t}$ and the definitions (7.1)-(7.3) we can directly verify that

$$
\left(\partial_{\tau}^{2}\left(\varepsilon \mathbf{E}_{h}^{m}-\varepsilon \nabla \chi_{h}^{m}\right), \nabla \varphi_{h}\right)=\left\langle\partial_{\tau}^{2} \rho^{m}-\rho_{t t}^{m}, \varphi_{h}\right\rangle, \quad \forall \varphi_{h} \in U_{h}
$$

for $m=1,2, \cdots, M$, which implies that

$$
\begin{aligned}
\left(\partial_{\tau}\left(\varepsilon \mathbf{E}_{h}^{m}-\varepsilon \nabla \chi_{h}^{m}\right), \nabla \varphi_{h}\right) & =\tau \sum_{j=1}^{m}\left(\partial_{\tau}^{2}\left(\varepsilon \mathbf{E}_{h}^{j}-\varepsilon \nabla \chi_{h}^{j}\right), \nabla \varphi_{h}\right)+\left(\partial_{\tau}\left(\varepsilon \mathbf{E}_{h}^{0}-\varepsilon \nabla \chi_{h}^{0}\right), \nabla \varphi_{h}\right) \\
& =\tau \sum_{j=1}^{m}\left\langle\partial_{\tau}^{2} \rho^{j}-\rho_{t t}^{j}, \varphi_{h}\right\rangle+\left(\varepsilon \mathbf{F}_{h}^{0}-\varepsilon \nabla \theta_{h}^{0}, \nabla \varphi_{h}\right),
\end{aligned}
$$

hence

$$
\begin{aligned}
& \left(\varepsilon \mathbf{E}_{h}^{m}-\varepsilon \nabla \chi_{h}^{m}, \nabla \varphi_{h}\right)=\tau \sum_{i=1}^{m}\left(\partial_{\tau}\left(\varepsilon \mathbf{E}_{h}^{i}-\varepsilon \nabla \chi_{h}^{i}\right), \nabla \varphi_{h}\right)+\left(\varepsilon \mathbf{E}_{h}^{0}-\varepsilon \nabla \chi_{h}^{0}, \nabla \varphi_{h}\right) \\
& =\tau^{2} \sum_{i=1}^{m} \sum_{j=1}^{i}\left\langle\partial_{\tau}^{2} \rho^{j}-\rho_{t t}^{j}, \varphi_{h}\right\rangle+m \tau\left(\varepsilon \mathbf{F}_{h}^{0}-\varepsilon \nabla \theta_{h}^{0}, \nabla \varphi_{h}\right)+\left(\varepsilon \mathbf{E}_{h}^{0}-\varepsilon \nabla \chi_{h}^{0}, \nabla \varphi_{h}\right) .
\end{aligned}
$$

That is,

$$
\left(\varepsilon \mathbf{E}_{h}^{m}-\varepsilon \nabla \chi_{h}^{m}-\left(\varepsilon \mathbf{E}_{h}^{0}-\varepsilon \nabla \chi_{h}^{0}\right)-m \tau\left(\varepsilon \mathbf{F}_{h}^{0}-\varepsilon \nabla \theta_{h}^{0}\right), \nabla \varphi_{h}\right)=\tau^{2} \sum_{i=1}^{m} \sum_{j=1}^{i}\left\langle\partial_{\tau}^{2} \rho^{j}-\rho_{t t}^{j}, \varphi_{h}\right\rangle .
$$

Then we can apply Lemma 7.1 for $1 / 2<s \leq 1$ to obtain

$$
\begin{aligned}
& \left\|\operatorname{div}\left(\varepsilon \mathbf{E}_{h}^{m}\right)-\operatorname{div}\left(\varepsilon \nabla \chi_{h}^{m}\right)\right\|_{-s} \\
& \lesssim\left\|\operatorname{div}\left(\varepsilon \mathbf{E}_{h}^{0}\right)-\operatorname{div}\left(\varepsilon \nabla \chi_{h}^{0}\right)\right\|_{-s}+m \tau\left\|\operatorname{div}\left(\varepsilon \mathbf{F}_{h}^{0}\right)-\operatorname{div}\left(\varepsilon \nabla \theta_{h}^{0}\right)\right\|_{-s} \\
& \quad+h^{s+\delta-1}\left\|\operatorname{curl}\left(\mathbf{E}_{h}^{m}-\mathbf{E}_{h}^{0}-m \tau \mathbf{F}_{h}^{0}\right)\right\|_{0}+\tau^{2} \sum_{i=1}^{m} \sum_{j=1}^{i}\left\|\partial_{\tau}^{2} \rho^{j}-\rho_{t t}^{j}\right\|_{-s} .
\end{aligned}
$$

We are now going to estimate the four terms on the right hand side of (7.12). Similarly to (4.19) and (4.21), we can derive from (7.2)-(7.4) that

$$
\begin{aligned}
\left\|\operatorname{div}\left(\varepsilon \mathbf{E}_{h}^{0}\right)-\operatorname{div}\left(\varepsilon \nabla \chi_{h}^{0}\right)\right\|_{-s} & \lesssim h^{s+\delta-1}\left\|\operatorname{curl}_{h}^{0}\right\|_{0} \lesssim h^{s+\delta-1}\left(\left\|\operatorname{curl} \mathbf{E}^{0}\right\|_{0}+\left\|\rho^{0}\right\|_{-1}\right), \\
\left\|\operatorname{div}\left(\varepsilon \mathbf{F}_{h}^{0}\right)-\operatorname{div}\left(\varepsilon \nabla \theta_{h}^{0}\right)\right\|_{-s} & \lesssim h^{s+\delta-1}\left\|\operatorname{curl}_{h}^{0}\right\|_{0} \lesssim h^{s+\delta-1}\left(\left\|\operatorname{curl} \mathbf{F}^{0}\right\|_{0}+\left\|\rho_{t}^{0}\right\|_{-1}\right) .
\end{aligned}
$$

Next we estimate $\left\|\operatorname{curl} \mathbf{E}_{h}^{m}\right\|_{0}$. Taking $\mathbf{v}_{h}=\tau \partial_{\tau} \mathbf{E}_{h}^{m}$ in (7.1) we come to

$$
\left(\varepsilon\left(\partial_{\tau} \mathbf{E}_{h}^{m}-\partial_{\tau} \mathbf{E}_{h}^{m-1}\right), \partial_{\tau} \mathbf{E}_{h}^{m}\right)+\left(\mu^{-1} \operatorname{curl} \mathbf{E}_{h}^{m}, \operatorname{curl}\left(\mathbf{E}_{h}^{m}-\mathbf{E}_{h}^{m-1}\right)\right)=\tau\left(\mathbf{f}^{m}, \partial_{\tau} \mathbf{E}_{h}^{m}\right)
$$


for $m=1,2, \cdots, M$. This implies readily that

$$
\begin{aligned}
\left\|\partial_{\tau} \mathbf{E}_{h}^{m}\right\|_{0, \varepsilon}^{2}+\left\|\operatorname{curl} \mathbf{E}_{h}^{m}\right\|_{0, \mu^{-1}}^{2} \leq & \tau\left\|\mathbf{f}^{m}\right\|_{0, \varepsilon^{-1}}\left\|\partial_{\tau} \mathbf{E}_{h}^{m}\right\|_{0, \varepsilon}+\left\|\partial_{\tau} \mathbf{E}_{h}^{m}\right\|_{0, \varepsilon}\left\|\partial_{\tau} \mathbf{E}_{h}^{m-1}\right\|_{0, \varepsilon} \\
& +\left\|\mathbf{c u r l} \mathbf{E}_{h}^{m}\right\|_{0, \mu^{-1}}\left\|\operatorname{curl} \mathbf{E}_{h}^{m-1}\right\|_{0, \mu^{-1}} \\
\leq & \frac{\tau}{2(1+\tau)}\left\|\partial_{\tau} \mathbf{E}_{h}^{m}\right\|_{0, \varepsilon}^{2}+\frac{\tau(1+\tau)}{2}\left\|\mathbf{f}^{m}\right\|_{0, \varepsilon^{-1}}^{2} \\
& +\frac{1}{2(1+\tau)}\left\|\partial_{\tau} \mathbf{E}_{h}^{m}\right\|_{0, \varepsilon}^{2}+\frac{1+\tau}{2}\left\|\partial_{\tau} \mathbf{E}_{h}^{m-1}\right\|_{0, \varepsilon}^{2} \\
& +\frac{1}{2}\left\|\mathbf{c u r l} \mathbf{E}_{h}^{m}\right\|_{0, \mu^{-1}}^{2}+\frac{1}{2}\left\|\mathbf{c u r l} \mathbf{E}_{h}^{m-1}\right\|_{0, \mu^{-1}}^{2} .
\end{aligned}
$$

Therefore,

$$
\left\|\partial_{\tau} \mathbf{E}_{h}^{m}\right\|_{0, \varepsilon}^{2}+\left\|\mathbf{c u r l} \mathbf{E}_{h}^{m}\right\|_{0, \mu^{-1}}^{2} \leq(1+\tau)\left(\left\|\partial_{\tau} \mathbf{E}_{h}^{m-1}\right\|_{0, \varepsilon}^{2}+\left\|\operatorname{curl} \mathbf{E}_{h}^{m-1}\right\|_{0, \mu^{-1}}^{2}\right)+\tau(1+\tau)\left\|\mathbf{f}^{m}\right\|_{0, \varepsilon^{-1}}^{2} .
$$

Using the above estimate recursively we obtain

$$
\begin{aligned}
\left\|\partial_{\tau} \mathbf{E}_{h}^{m}\right\|_{0, \varepsilon}^{2}+\left\|\operatorname{curl} \mathbf{E}_{h}^{m}\right\|_{0, \mu^{-1}}^{2} & \lesssim\left\|\partial_{\tau} \mathbf{E}_{h}^{0}\right\|_{0, \varepsilon}^{2}+\left\|\operatorname{curl} \mathbf{E}_{h}^{0}\right\|_{0, \mu^{-1}}^{2}+\tau \sum_{j=1}^{m}\left\|\mathbf{f}^{j}\right\|_{0, \varepsilon^{-1}}^{2} \\
& \lesssim\left\|\mathbf{F}_{h}^{0}\right\|_{0}^{2}+\left\|\operatorname{curl} \mathbf{E}_{h}^{0}\right\|_{0}^{2}+\tau \sum_{j=1}^{m}\left\|\mathbf{f}^{j}\right\|_{0}^{2}
\end{aligned}
$$

On the other hand, it follows from (7.3), (7.4) and (7.14), and the argument for the $L^{2}$-error estimates (cf. Lemma 4.4-(4.21) for small $h$ ) that

$$
\begin{aligned}
\left\|\mathbf{F}_{h}^{0}\right\|_{0} & \lesssim\left\|\mathbf{F}^{0}\right\|_{0}+\left\|\mathbf{F}_{h}^{0}-\mathbf{F}^{0}\right\|_{0} \lesssim\left\|\mathbf{F}^{0}\right\|_{\mathbf{H}(\operatorname{curl} ; \Omega)}+\left\|\rho_{t}^{0}\right\|_{-1}+\left\|\operatorname{div}\left(\varepsilon \mathbf{F}^{0}-\varepsilon \mathbf{F}_{h}^{0}\right)\right\|_{-1} \\
& \lesssim\left\|\mathbf{F}^{0}\right\|_{\mathbf{H}(\operatorname{curl} ; \Omega)}+\left\|\rho_{t}^{0}\right\|_{-1}+\left\|\operatorname{div}\left(\varepsilon \mathbf{F}^{0}-\varepsilon \nabla \theta_{h}^{0}\right)\right\|_{-1}+\left\|\operatorname{div}\left(\varepsilon \nabla \theta_{h}^{0}-\varepsilon \mathbf{F}_{h}^{0}\right)\right\|_{-1} \\
& \lesssim\left\|\mathbf{F}^{0}\right\|_{\mathbf{H}(\operatorname{curl} ; \Omega)}+\left\|\rho_{t}^{0}\right\|_{-1} .
\end{aligned}
$$

Hence we obtain from the previous two estimates

$$
\left\|\operatorname{curl} \mathbf{E}_{h}^{m}\right\|_{0}^{2} \lesssim\left\|\mathbf{F}^{0}\right\|_{\mathbf{H}(\operatorname{curl} ; \Omega)}^{2}+\left\|\operatorname{curl} \mathbf{E}^{0}\right\|_{0}^{2}+\left\|\rho^{0}\right\|_{-1}^{2}+\left\|\rho_{t}^{0}\right\|_{-1}^{2}+\sum_{j=1}^{m} \tau\left\|\mathbf{f}^{j}\right\|_{0}^{2} .
$$

Finally we come to estimate $\left\|\partial_{\tau}^{2} \rho^{j}-\rho_{t t}^{j}\right\|_{-s}$. Using the Taylor expansion with integral remainder, we can easily deduce that

$$
\begin{aligned}
& \left\|\partial_{\tau}^{2} \rho^{j}-\rho_{t t}^{j}\right\|_{-s} \lesssim \int_{t^{j-2}}^{t^{j}}\left\|\rho_{t t t}\right\|_{-s}, \quad j>1, \\
& \left\|\partial_{\tau}^{2} \rho^{1}-\rho_{t t}^{1}\right\|_{-s}=\left\|\frac{\rho^{1}-\rho^{0}-\tau \rho_{t}^{0}}{\tau^{2}}-\rho_{t t}^{1}\right\|_{-s} \lesssim\left\|\rho_{t t}^{0}\right\|_{-s}+\int_{0}^{\tau}\left\|\rho_{t t t}\right\|_{-s} .
\end{aligned}
$$

Therefore

$$
\tau^{2} \sum_{i=1}^{m} \sum_{j=1}^{i}\left\|\partial_{\tau}^{2} \rho^{j}-\rho_{t t}^{j}\right\|_{-s} \lesssim \tau\left(\left\|\rho_{t t}^{0}\right\|_{-s}+\int_{0}^{t^{m}}\left\|\rho_{t t t}\right\|_{-s}\right) .
$$

By combining (7.12)-(7.16), we have

$$
\begin{aligned}
& \left\|\operatorname{div}\left(\varepsilon \mathbf{E}_{h}^{m}\right)-\operatorname{div}\left(\varepsilon \nabla \chi_{h}^{m}\right)\right\|_{-s} \lesssim \tau\left(\left\|\rho_{t t}^{0}\right\|_{-s}+\int_{0}^{t^{m}}\left\|\rho_{t t t}\right\|_{-s}\right) \\
& \quad+h^{s+\delta-1}\left(\left\|\mathbf{F}^{0}\right\|_{\mathbf{H}(\mathbf{c u r l} ; \Omega)}+\left\|\mathbf{c u r l} \mathbf{E}^{0}\right\|_{0}+\left\|\rho^{0}\right\|_{-1}+\left\|\rho_{t}^{0}\right\|_{-1}+\left(\sum_{j=1}^{m} \tau\left\|\mathbf{f}^{j}\right\|_{0}^{2}\right)^{1 / 2}\right) .
\end{aligned}
$$


On the other hand, we know from Lemma 3.9 that

$$
\left\|\operatorname{div}\left(\varepsilon \mathbf{E}^{m}\right)-\operatorname{div}\left(\varepsilon \nabla \chi_{h}^{m}\right)\right\|_{-s} \lesssim h^{s+\delta-1}\left\|\rho^{m}\right\|_{\delta-1} .
$$

Now the desired error estimate (7.6) for the divergence is a direct consequence of (7.17)-(7.18).

\section{Numerical examples}

In this section we present a numerical example to confirm the optimal convergence rate of the edge element scheme (4.4) for solving the stationary Maxwell system (1.1)-(1.2). We take the domain $\Omega=$ $(0,1) \times(0,1) \times(0,1)$, and the coefficients $\varepsilon=1$ and $\mu=1$. Functions $\mathbf{f}$ and $\rho$ are chosen such that the exact solution $\mathbf{E}$ to the system (1.1)-(1.2) is given by

$$
\mathbf{E}=\left(\begin{array}{l}
x_{1} x_{2} x_{3}\left(1-x_{2}\right)\left(1-x_{3}\right) \\
x_{1} x_{2} x_{3}\left(1-x_{3}\right)\left(1-x_{1}\right) \\
x_{1} x_{2} x_{3}\left(1-x_{1}\right)\left(1-x_{2}\right)
\end{array}\right)
$$

As the domain $\Omega$ is convex, we have the regularity exponents $\delta=\delta^{\prime}=1$ in Theorem 4.2 . So we have the following error estimates for $1 / 2<s \leq 1$ :

$$
\left\|\mathbf{E}-\mathbf{E}_{h}\right\|_{0}=\left\|\operatorname{curl}\left(\mathbf{E}-\mathbf{E}_{h}\right)\right\|_{0}=O(h), \quad\left\|\operatorname{div} \mathbf{E}-\operatorname{div} \mathbf{E}_{h}\right\|_{-s}=O\left(h^{s}\right) .
$$

We start with a uniform initial triangulation $\mathcal{T}_{0}$ of $\Omega$, consisting of 192 tetrahedra. Then we refine $\mathcal{T}_{0}$ successively and obtain the triangulation $\mathcal{T}_{j}$ at the $j$ th refinement with mesh size $h_{j}$. The refinements are done in such a uniform way that each tetrahedron in $\mathcal{T}_{j-1}$ is divided into eight sub-tetrahedra to generate the mesh $\mathcal{T}_{j}$. Clearly we have $h_{j}=2^{-j} h_{0}$. We set $\gamma\left(h_{j}\right)=h_{j}^{2}$ unless otherwise specified. We use the software COMSOL Multiphysics for our experiments and write our codes in MATLAB.

In our experiments, we solve:

- the system (3.16) for the solution $\chi_{h}$ (required in the edge element approximation (4.4)) by the preconditioned CG method with the multigrid preconditioner;

- the edge element system (4.4) by the preconditioned bi-CG stabilized method (cf. [34]) with the Hiptmair-Xu preconditioner (cf. [22]).

We first check the $L^{2}$-norm and curl-norm error estimates in (8.2). The errors $\left\|\mathbf{E}-\mathbf{E}_{h_{j}}\right\|_{0}$ (dashed) and $\left\|\operatorname{curl}\left(\mathbf{E}-\mathbf{E}_{h_{j}}\right)\right\|_{0}\left(\right.$ solid) are plotted in Figure 1 against $1 / h_{j}$ in $\log$ - $\log$ scale for $j=0,1, \cdots 5$. Clearly this numerical example has verified very well the predicted error estimates:

$$
\left\|\mathbf{E}-\mathbf{E}_{h_{j}}\right\|_{0} \simeq h_{j}, \quad\left\|\operatorname{curl}\left(\mathbf{E}-\mathbf{E}_{h_{j}}\right)\right\|_{0} \simeq h_{j}
$$

Secondly we test the convergence rate of $\left\|\operatorname{div} \mathbf{E}-\operatorname{div} \mathbf{E}_{h}\right\|_{-s}$ for $1 / 2<s \leq 1$. Although $\operatorname{div} \mathbf{E}_{h}$ vanishes in every $K \in \mathcal{T}_{h}$, the "global" (weak) divergence of $\mathbf{E}_{h}$ does not. Theoretically, we know directly from the proofs of Lemma 3.8 and Theorem 4.2 the following error estimate

$$
\left\|\operatorname{div} \mathbf{E}-\operatorname{div} \mathbf{E}_{h}\right\|_{-s} \lesssim h^{s}\|\rho\|_{0}+h^{s-1 / 2}\left(\sum_{f \in \mathcal{F}_{h}}\left\|\left[\left[\mathbf{E}_{h} \cdot \mathbf{n}\right]\right]\right\|_{L^{2}(f)}^{2}\right)^{1 / 2}
$$

for $\rho \in L^{2}(\Omega)$ and $1 / 2<s \leq 1$, where $\left[\left[\mathbf{E}_{h} \cdot \mathbf{n}\right]\right]$ are the jumps over element faces $f \in \mathcal{F}_{h}$ as defined in (3.25). We emphasize that the estimate (8.3) holds without assuming that the triangulations are quasi-uniform, since only (3.26)-(3.27) are needed. If we introduce the estimator

$$
\eta_{h}=\left(\sum_{f \in \mathcal{F}_{h}}\left\|\left[\left[\mathbf{E}_{h} \cdot \mathbf{n}\right]\right]\right\|_{L^{2}(f)}^{2}\right)^{1 / 2}
$$

then it suffices to check that $\eta_{h}=O\left(h^{1 / 2}\right)$ in order to verify the convergence rate of the divergence given by (8.3). Indeed this can be very well confirmed; see Figure 2. 


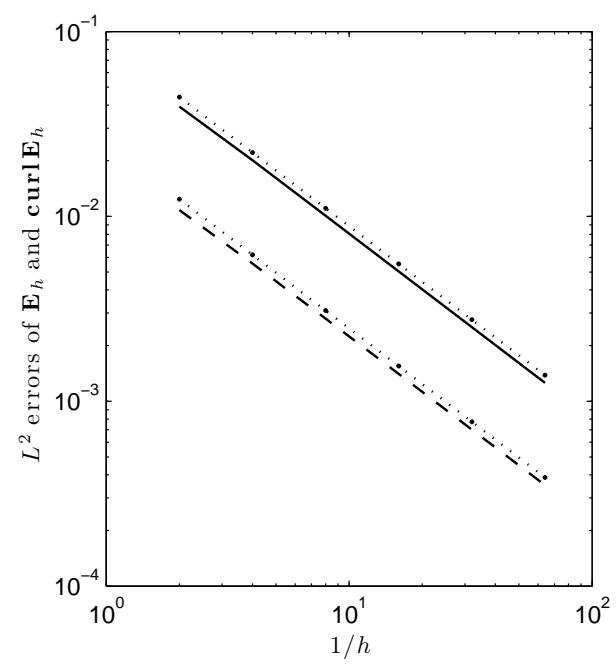

Figure 1: $\left\|\mathbf{E}-\mathbf{E}_{h_{j}}\right\|_{0}$ (dashed) and $\left\|\operatorname{curl}\left(\mathbf{E}-\mathbf{E}_{h_{j}}\right)\right\|_{0}$ (solid) versus the numbers of degrees of freedom in log-log scale. The dotted lines with dot markers gives the reference line with slope -1 . The markers correspond to $j=0,1, \cdots, 5$.

Next, we demonstrate the performance of the Hiptmair-Xu preconditioner for the edge element scheme (4.4). Table 1 shows the number of iterations of the preconditioned bi-CG stabilized method for solving (4.4). In our experiments, the tolerance is set to be $10^{-8}$ for the relative $l^{2}$-norm of the residual. We recall that the condition number of the preconditioned system of (4.4) by the Hiptmair-Xu preconditioner is independent of the parameter $\gamma(h)$ and the multilevel $j$ (cf. [22]). Clearly this is very well confirmed in Table 1. In fact, the preconditioned bi-CG stabilized method converges optimally, namely one iteration of the method can reduce the norm of the error of the approximate solution by a factor that is bounded away from 1 and independent of $N$, the size of the linear system, resulting in a total computational complexity of order $O(N)$.

\begin{tabular}{|c|c|c|c|c|c|}
\hline$j$ & 1 & 2 & 3 & 4 & 5 \\
\hline$N$ & 1,516 & 13,208 & 110,128 & 899,168 & $7,266,496$ \\
\hline$\gamma\left(h_{j}\right)$ & $1 / 16$ & $1 / 64$ & $1 / 256$ & $1 / 1024$ & $1 / 4096$ \\
\hline Iter & 4.5 & 6.25 & 8.5 & 10 & 9 \\
\hline
\end{tabular}

Table 1: Number of refinements, number of degrees of freedom of the linear system, the parameter $\gamma\left(h_{j}\right)=h_{j}^{2}$, and number of iterations of the multigrid preconditioned bi-stabilized method.

Finally, we test the possible influence of round-off errors when $\gamma(h)$ is very small. Theoretically, the parameter $\gamma(h)$ can be chosen arbitrarily small (but positive) without affecting the convergence orders and the performance of the preconditioned iterative methods, but numerically, the actual effect of the perturbation terms involving $\gamma(h)$ may be affected by the round-off errors if $\gamma(h)$ is too small. We fix $j=3$ and set the tolerance to be $10^{-10}$. Table 2 shows that the errors in $H($ curl $)$-norm and the number of iterations of the multigrid preconditioned bi-stabilized method behave well for $\gamma\left(h_{3}\right)$ up to $10^{-8}$, while the errors in $L^{2}$-norm and the estimator $\eta_{h}$ remain steady for $\gamma\left(h_{3}\right)$ up to $10^{-7}$.

Acknowledgments. The authors would like to thank the anonymous referees for their many insightful and constructive comments and suggestions that have well improved the quality of the paper.

\section{References}

[1] H. Ammari, An Introduction to Mathematics of Emerging Biomedical Imaging, Mathématiques et Applications, Vol. 62, Springer-Verlag, Berlin, 2008. 


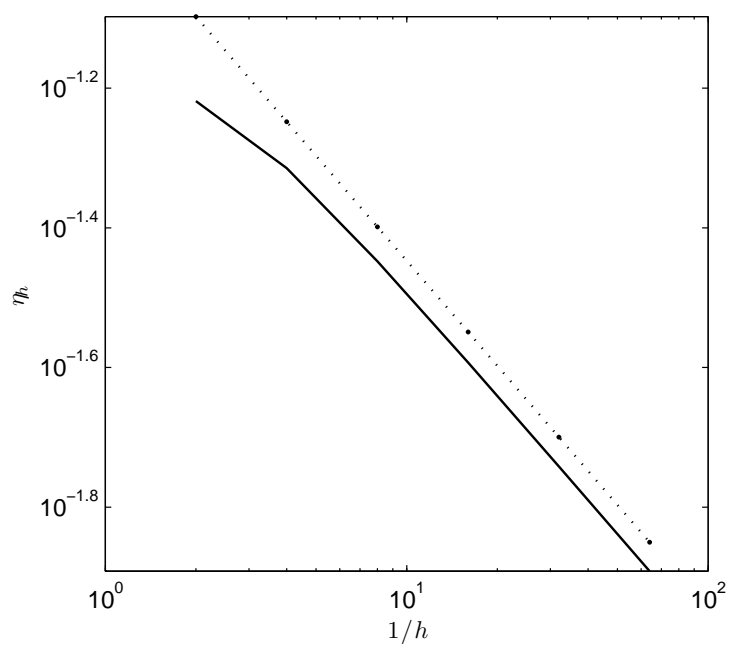

Figure 2: $\eta_{h_{j}}$ versus $1 / h_{j}$ in log-log scale (solid line). The dotted line with dot markers gives the reference line with slope $-1 / 2$. The markers correspond to $j=0,1, \cdots, 5$.

\begin{tabular}{|c|c|c|c|c|c|c|}
\hline$\gamma\left(h_{3}\right)$ & $10^{-3}$ & $10^{-4}$ & $10^{-5}$ & $10^{-6}$ & $10^{-7}$ & $10^{-8}$ \\
\hline$\|$ curl $\left(\mathbf{E}-\mathbf{E}_{h_{3}}\right) \|_{0}$ & $5.0 \times 10^{-3}$ & $5.0 \times 10^{-3}$ & $5.0 \times 10^{-3}$ & $5.0 \times 10^{-3}$ & $5.0 \times 10^{-3}$ & $5.0 \times 10^{-3}$ \\
\hline$\left\|\mathbf{E}-\mathbf{E}_{h_{3}}\right\|_{0}$ & $1.4 \times 10^{-3}$ & $1.4 \times 10^{-3}$ & $1.4 \times 10^{-3}$ & $1.4 \times 10^{-3}$ & $1.4 \times 10^{-3}$ & $4.4 \times 10^{-3}$ \\
\hline$\eta_{h_{3}}$ & $2.6 \times 10^{-2}$ & $2.6 \times 10^{-2}$ & $2.6 \times 10^{-2}$ & $2.6 \times 10^{-2}$ & $2.6 \times 10^{-2}$ & $3.9 \times 10^{-2}$ \\
\hline Iter & 13.25 & 17 & 19 & 17.25 & 12.5 & 5 \\
\hline
\end{tabular}

Table 2: Influence of $\gamma(h)$ on the errors of the discrete solution and the number of iterations of the multigrid preconditioned bi-stabilized method.

[2] H. Ammari And H. KAng, Reconstruction of Small Inhomogeneities from Boundary Measurements, Lecture Notes in Mathematics, Vol. 1846, Springer-Verlag, Berlin, 2004.

[3] C. Amrouche, C. Bernardi, M. Dauge and V. Girault, Vector potentials in three-dimensional non-smooth domains, Math. Meth. Appl. Sci. 21 (1998), 823-864.

[4] R. Bank, B. Welfert, and H. Yserentant, A class of iterative methods for solving saddle point problems, Numer. Math. 56 (1990), 645-666.

[5] R. Barthelmé, P. Ciarlet, Jr. And E. Sonnendrǘcker, Generalized formulations of Maxwell's equations for numerical Vlasov-Maxwell simulations, Math. Mod. Meth. Appl. Sci. 17 (2007), 657680 .

[6] J. Bergh And J. LÖFström, Interpolation Spaces: an Introduction, Springer Verlag, 1976.

[7] A. Bonito And J.-L. Guermond, Approximation of the eigenvalue problem for the time harmonic Maxwell system by continuous Lagrange finite elements, Math. Comp. 80 (2011), 1887-1910.

[8] S.C. Brenner And L.R. ScotT The Mathematical Theory of Finite Element Methods, 3rd Edition, Springer Verlag, 2008.

[9] F. Brezzi And M. Fortin Mixed and hybrid finite element methods, Springer Series in Computational Mathematics 15, Springer, 1991.

[10] Z. Chen, Q. Du And J. Zou, Finite element methods with matching and nonmatching meshes for Maxwell equations with discontinuous coefficients, SIAM J. Numer. Anal. 37 (2000), 1542-1570.

[11] Z. Chen And H. Wu, Selected Topics in Finite Element Methods, Science Press, 2010. 
[12] P. Ciarlet, JR, T-coercivity: Application to the discretization of Helmholtz-like problems, Comput. Math. Appl. 64 (2012), 22-34.

[13] P. Ciarlet, JR, Analysis of the Scott-Zhang interpolation in the fractional order Sobolev spaces, J. Numer. Math. 21 (2013), 173-180.

[14] P. Ciarlet, Jr. and S. Labrunie, Numerical analysis of the generalized Maxwell equations (with an elliptic correction) for charged particle simulations, Math. Mod. Meth. Appl. Sci. 19 (2009), 1959-1994.

[15] P. Ciarlet, Jr. and J. Zou, Finite element convergence for the Darwin model to Maxwell's equations, Math. Mod. Num. Anal. 31 (1997), 213-250.

[16] P. Ciarlet, Jr. And J. Zou, Fully discrete finite element approaches for time-dependent Maxwell's equations, Numer. Math. 82 (1999), 193-219.

[17] M. Costabel, M. Dauge and S. Nicaise, Singularities of Maxwell interface problems, Math. Mod. Num. Anal. 33 (1999), 627-649.

[18] M. Dauge, Elliptic Boundary Value Problems on Corner Domains, Lecture notes in mathematics 1341, Springer, 1988.

[19] M. Dauge, Singularities of corner problems and problems of corner singularities, ESAIM: Proceedings 6 (1999), 19-40.

[20] V. Girault and P.A. Raviart Finite element methods for Navier-Stokes equations, Springer Series in Computational Mathematics 5, Springer, 1986.

[21] R. Hiptmair, Finite elements in computational electromagnetism, Acta Numerica 11 (2002), 237339.

[22] R. Hiptmair and J. Xu, Nodal auxiliary space preconditioning in $\mathbf{H}($ curl ) and $\mathbf{H}($ div ) spaces, SIAM J. Numer. Anal. 45 (2007), 2483-2509.

[23] Q. Hu And J. Zou, Substructuring preconditioners for saddle-point problems arising from Maxwell's equations in three dimensions, Math. Comput. 73 (2004), 35-61.

[24] Q. Hu And J. Zou, Nonlinear inexact Uzawa algorithms for linear and nonlinear saddle-point problems, SIAM J. Optimiz. 16 (2006) , 798-825.

[25] Q. Hu, S. Shu And J. Zou, A mortar edge element method with nearly optimal convergence for three-dimensional Maxwell's equations, Math. Comput. 77 (2008), 1333-1353.

[26] D. Jerison And C. E Kenig, The inhomogeneous Dirichlet problem in Lipschitz domains, J. Funct. Anal. 130 (1995), 161-219.

[27] A. B. Langdon, On enforcing Gauss' law in electromagnetic particle-in-cell codes, Comput. Phys. Commun. 70 (1992), 447-450.

[28] B. Marder, A method for incorporating Gauss's law into electromagnetic PIC codes, J. Comput. Phys. 68 (1987), 48-55.

[29] P. Monk, Finite Element Methods for Maxwell's quations, Oxford University Press, USA, 2003.

[30] J. NÉDÉLEC, Mixed finite elements in $\mathbb{R}^{3}$, Numer. Math. 35 (1980), 315-341.

[31] S. Nicaise And A.-M. SÄndig, Transmission problems for the Laplace and elasticity operators: regularity and boundary integral formulation, Math. Models Meth. App. Sci. 9 (1999), 855-898.

[32] T. Rusten and R. Winther, A preconditioned iterative method for saddlepoint problems, SIAM J. Matrix Anal. Appl. 13 (1992), 887-904. 
[33] L.R. Scott and S. Zhang, Finite element interpolation of nonsmooth functions satisfying boundary conditions, Math. Comp. 54 (1990), 483-493.

[34] G. Sleijpen, H. van der Vorst and D. Fokkema, BiCGstab(ell) and other Hybrid Bi-CG methods, Numer. Alg. 7 (1994), 75-109. 